\title{
Safety Evaluation of $\alpha$-Lipoic Acid Supplementation: A Systematic Review and Meta-Analysis of Randomized Placebo-Controlled Clinical Studies
}

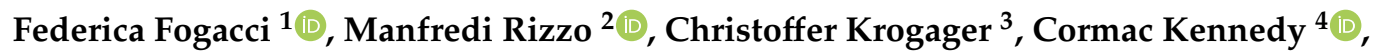 \\ Coralie M.G. Georges ${ }^{5}$, Tamara Knežević ${ }^{6}$, Evangelos Liberopoulos ${ }^{7}{ }^{\circ}$, Alexandre Vallée ${ }^{8}(\mathbb{D}$, \\ Pablo Pérez-Martínez ${ }^{9,10,11,12}$, Eliane F.E. Wenstedt ${ }^{13}$, Agnè Šatrauskienè ${ }^{14,15}$, \\ Michal Vrablík ${ }^{16}\left(\mathbb{D}\right.$ and Arrigo F.G. Cicero ${ }^{1, *(1)}$
}

1 Hypertension and Cardiovascular Risk Factors Research Group, Medical and Surgical Sciences Department, Sant'Orsola-Malpighi University Hospital, 40138 Bologna, Italy; federica.fogacci@studio.unibo.it

2 Department of Health Promotion Sciences Maternal and Infantile Care, Internal Medicine and Medical Specialities (PROMISE), School of Medicine, University of Palermo, 90127 Palermo, Italy; manfredi.rizzo@unipa.it

3 Department of Endocrinology, Aarhus University Hospital, DK-8200 Aarhus N, Denmark; chrkroga@rm.dk

4 Department of Pharmacology and Therapeutics, Trinity College Dublin and St James Hospital, Dublin 8, Ireland; kennec30@tcd.ie

5 Department of Cardiology, Cliniques Universitaires Saint-Luc, Université Catholique de Louvain, 1200 Brussels, Belgium; coralie.georges@uclouvain.be

6 Department of Nephrology, Hypertension, Dialysis and Transplantation, University Hospital Centre Zagreb, 10000 Zagreb, Croatia; tknezev2@kbc-zagreb.hr

7 Faculty of Medicine, School of Health Sciences, University of Ioannina, 45110 Ioannina, Greece; elibero@uoi.gr

8 Diagnosis and Therapeutic Center, Hôtel-Dieu Hospital, Paris-Descartes University, 75004 Paris, France; alexandre.g.vallee@gmail.com

9 CIBER Fisiopatología de la Obesidad y Nutrición (CIBEROBN), Instituto de Salud Carlos III (ISCIII), 28007 Madrid, Spain; pabloperez@uco.es

10 Lipids and Atherosclerosis Unit, Department of Internal Medicine, Reina Sofia University Hospital, 14004 Cordoba, Spain

11 Maimonides Biomedical Research Institute of Cordoba (IMIBIC), 14004 Cordoba, Spain

12 Department of Medicine (Medicine, Dermatology and Otorhinolaryngology), University of Cordoba, 14004 Cordoba, Spain

13 Amsterdam UMC-University of Amsterdam, 1100 DD Amsterdam, The Netherlands; elianewenstedt@live.nl

14 Faculty of Medicine, Vilnius University, LT-03101 Vilnius, Lithuania; agne.satrauskiene@santa.it

15 Vilnius University Hospital Santariškiụ Klinikos, LT-08661 Vilnius, Lithuania

16 Third Department of Internal Medicine, First Medical Faculty, Charles University,

12808 Prague 2, Czech Republic; vrablikm@seznam.cz

* Correspondence: arrigo.cicero@unibo.it; Tel.: +39-512-142-224

Received: 19 September 2020; Accepted: 15 October 2020; Published: 19 October 2020

Abstract: Alpha-lipoic acid (ALA) is a natural short-chain fatty acid that has attracted great attention in recent years as an antioxidant molecule. However, some concerns have been recently raised regarding its safety profile. To address the issue, we aimed to assess ALA safety profile through a systematic review of the literature and a meta-analysis of the available randomized placebo-controlled clinical studies. The literature search included EMBASE, PubMed Medline, SCOPUS, Google Scholar, and ISI Web of Science by Clarivate databases up to 15th August 2020. Data were pooled from 71 clinical studies, comprising 155 treatment arms, which included 4749 subjects with 2558 subjects treated with ALA and 2294 assigned to placebo. A meta-analysis of extracted data suggested that supplementation with ALA was not associated with an increased risk of any treatment-emergent adverse event 
(all $p>0.05$ ). ALA supplementation was safe, even in subsets of studies categorized according to smoking habit, cardiovascular disease, presence of diabetes, pregnancy status, neurological disorders, rheumatic affections, severe renal impairment, and status of children/adolescents at baseline.

Keywords: $\alpha$-lipoic acid; thioctic acid; dietary supplement; safety; meta-analysis

\section{Introduction}

Alpha-lipoic acid (1, 2-dithiolane-3-pentanoic acid; ALA) or thioctic acid is a natural short-chain fatty acid that has attracted great attention in recent years as an antioxidant molecule, being largely used worldwide as a dietary supplement [1].

Previous investigations revealed that ALA can affect central and peripheral modulation of $5^{\prime}$-adenosine-monophosphate-activated protein kinase. Furthermore, it activates peroxisome proliferator-activated receptor (PPAR) alpha and gamma (PPAR- $\gamma$ ), modulates PPAR-regulated genes and upregulates the expression of PPAR- $\gamma$ messenger ribonucleic acid (mRNA) and other proteins in the cardiac tissue and aorta smooth muscle [2,3]. Hence, ALA antioxidant activity is potentially able to promote weight loss and blood pressure control and ameliorate atherogenic dyslipidemia and insulin resistance [3]. For example, in obese patients with non-alcoholic fatty liver disease (NAFLD), ALA supplementation was shown to reduce adipokine concentrations and improve liver steatosis grade $[4,5]$. However, some concerns have been recently raised regarding ALA safety profile, after some reports suggesting a direct causal link between its use and insulin autoimmune syndrome (IAS, also known as Hirata's disease) due to its sulfhydryl group [6]. Indeed, in about $50 \%$ of cases, IAS development is associated with drugs or dietary supplement containing a sulphur or sulfhydryl group. These cases are closely related to certain specific antigens of the major histocompatibility complex (MHC), which are more common in populations where IAS incidence is higher [7]. It is hypothesised that ALA might cause the development of antibodies to insulin and lead to a hypoglycaemic syndrome in predisposed subjects, even though evidence are inconclusive [8].

In a recent study that performed a preliminary analysis of spontaneous reports of suspected adverse reactions (ARs), ALA-containing natural products have also been associated with skin and gastrointestinal disorders, such as urticaria and abdominal pain [9].

To address safety issues related to ALA supplementation, we aimed to perform a systematic review of the literature and a meta-analysis of the available randomized placebo-controlled clinical trials.

\section{Materials and Methods}

The study was designed according to guidelines of the 2009 preferred reporting items for systematic reviews and meta-analysis (PRISMA) statement [10], and was registered in the PROSPERO database (Registration number CRD42020159028).

Due to the study design, neither Institutional Review Board (IRB) approval, nor patient informed consent were required. PRISMA Checklist was reported in supplementary file A.

\subsection{Search Strategy}

EMBASE, PubMed Medline, SCOPUS, Google Scholar and ISI Web of Science by Clarivate databases were searched, with no language restriction, using the following search terms: ("Alpha-lipoic acid" OR "Alpha lipoic acid" OR " $\alpha$-lipoic acid" OR " $\alpha$ lipoic acid" OR "ALA" OR "A-LA" OR “Lipoic acid" OR "Thioctic acid" OR "Tioctic acid" OR "Thioctacid") AND ("Clinical trial" OR "Clinical study"). The wild-card term "*" was used to increase the sensitivity of the search strategy, which was limited to studies in humans. The reference list of identified papers was manually checked for additional relevant articles. Additional searches included references of review articles on 
that issue, and abstracts from selected congresses on the subject of the meta-analysis. Literature was searched from inception to 15th August 2020.

All paper abstracts were firstly screened by two independent reviewers (F.F. and M.R.) to remove ineligible articles. The remaining articles were obtained in full-text and assessed again by the same two researchers who evaluated each article independently and carried out data extraction and quality assessment. Disagreements were resolved by discussion with a third party (A.F.G.C.).

\subsection{Study Selection Criteria}

Original studies were included if they met the following criteria: (i) being a clinical trial with either parallel or cross-over design, (ii) having an appropriate controlled design for ALA supplementation, (iii) blinding participants to intervention, (iv) testing the safety of ALA, (v) reporting treatment-emergent adverse events.

Exclusion criteria were: (i) lack of randomisation for treatment allocation, (ii) lack of a control group receiving placebo (iii) lack of sufficient information about the prevalence and nature of the adverse events. Studies were also excluded if they contained overlapping subjects with other studies.

\subsection{Data Extraction}

Data abstracted from eligible studies were: (i) first author's name; (ii) year of publication; (iii) study location; (iv) study design; (v) follow-up; (vi) main inclusion criteria and underlying disease; (vii) study groups; (viii) number of participants in the active and control group; (ix) age and sex of study participants; ( $x$ ) treatment-emergent adverse events occurred during the trials. Missing or unpublished data were sought by trying to contact authors via e-mail and repeated messages were sent in case of no response. Extracted data were reviewed by the principal investigator before the final analysis, and doubts were resolved by mutual agreement among the authors.

\subsection{Quality Assessment}

A systematic assessment of risk of bias in the included studies was performed using the Cochrane criteria [11]. The following items were used: adequacy of sequence generation, allocation concealment, blinding addressing of dropouts (incomplete outcome data), selective outcome reporting, and other probable sources of bias [12]. Overall evidence was qualified using the Grading of Recommendations, Assessment, Development, and Evaluations (GRADE) system [13]. Risk-of-bias assessment was performed independently by two reviewers; disagreements were resolved by a consensus-based discussion.

\subsection{Data Synthesis}

Meta-analysis was conducted using Comprehensive Meta-Analysis (CMA) V3 software (Biostat, NJ) [14].

Outcomes were treatment-emergent adverse events (AEs) occurring during the trials. In particular, data extracted from the studies included hypoglycaemic episodes, gastrointestinal AEs (e.g., heartburn, gastric complaints, nausea, gastrointestinal complications, duodenitis, and abdominal bloating), neurological AEs (e.g., headache, foggy thinking, drowsiness, leg weakness, legs periodic numbness and tingling, tingling in toe and fingers and intermittent bilateral toe numbness), psychiatric disorders (e.g., bipolar disorders, irritability, poor sleeping), musculoskeletal AEs (e.g., neck pain, lower back pain, and spasms), skin AEs (e.g., skin rash, disseminated maculopapular rash, itching sensation and urticaria), infections (e.g., laryngitis, pneumonia and yeast infections), cardiovascular (CV) system AEs (e.g., increase in arterial blood pressure, palpitations, myocardial infarction, heart rate and rhythm disorders, and heart valve disorders), hospitalisation and death.

The analysis was performed by excluding studies with zero events in both arms. If one or more outcomes could not be extracted from a study, the study was removed only from the analysis involving those outcomes. To avoid a double-counting problem, in trials comparing multiple treatment arms 
versus a single control group, the number of subjects in the control group was divided by the required comparisons [15].

To reduce the risk of bias due to effect dilution, the meta-analysis was performed considering per-protocol (PP) population.

Studies' findings were combined using a fixed-effect model since the low level of inter-study heterogeneity, which was quantitatively assessed using the Higgins index $\left(\mathrm{I}^{2}\right)$ [16]. Effect sizes were expressed as odds ratio (OR) and 95\% confidence interval (95\% CI) [17]. Finally, sensitivity analysis was conducted to account for the risk of bias. A leave-one-out method was used (i.e., one study was removed at a time and the analysis was repeated) [18].

Two-sided $p$-values $<0.05$ were considered as statistically significant for all tests.

\subsection{Additional Analysis}

Subgroup analyses were carried out by presence of smoking habit, pregnancy, CV disease, diabetes, rheumatic disorders, neurological disorders, severe renal impairment, and status of children/adolescent at baseline.

\subsection{Publication Biases}

Potential publication biases were explored using visual inspection of Begg's funnel plot asymmetry, Begg's rank correlation test, and Egger's weighted regression test [19]. Two-sided $p$-values $<0.05$ were considered statistically significant for the tests.

\section{Results}

\subsection{Flow and Characteristics of the Included Studies}

After database searches performed strictly according to inclusion and exclusion criteria, 962 published articles were identified, and their abstracts reviewed. Of these, 359 did not report original data. Furthermore, 393 articles were excluded because they did not meet the inclusion criteria. Thus, 210 articles were carefully assessed and reviewed. Additional 139 papers were excluded due to being pre-print papers $(n=2)$, study protocols $(n=6)$, reporting data from studies lacking of an appropriate placebo-controlled design for the supplementation $(n=64)$, lacking of randomisation $(n=5)$, testing the acute effect of ALA supplementation $(n=7)$, testing ALA supplementation combined in nutraceutical compounds ( $n=27)$, testing intravenous treatment with ALA $(n=11)$, testing topical treatment with ALA $(n=4)$, lacking sufficient information about the nature of the adverse events $(n=9)$, or reporting data overlapped with other publications $(n=4)$ (Supplementary file B). Finally, 71 studies were eligible and included in the systematic review [20-90]. The study selection process is shown in Figure 1.

Data were pooled from 71 randomized placebo-controlled clinical studies, comprising 155 treatment arms (82 active arms and 73 control arms). The studies included 4749 subjects, with 2558 receiving treatment with ALA and 2294 subjects assigned to placebo. For reasons independent of the tested supplementation (i.e., withdrawal of informed consent and personal problems), 510 subjects prematurely terminated the trials in which they were enrolled. Then, the meta-analysis was performed considering the other subjects (i.e., PP population). 


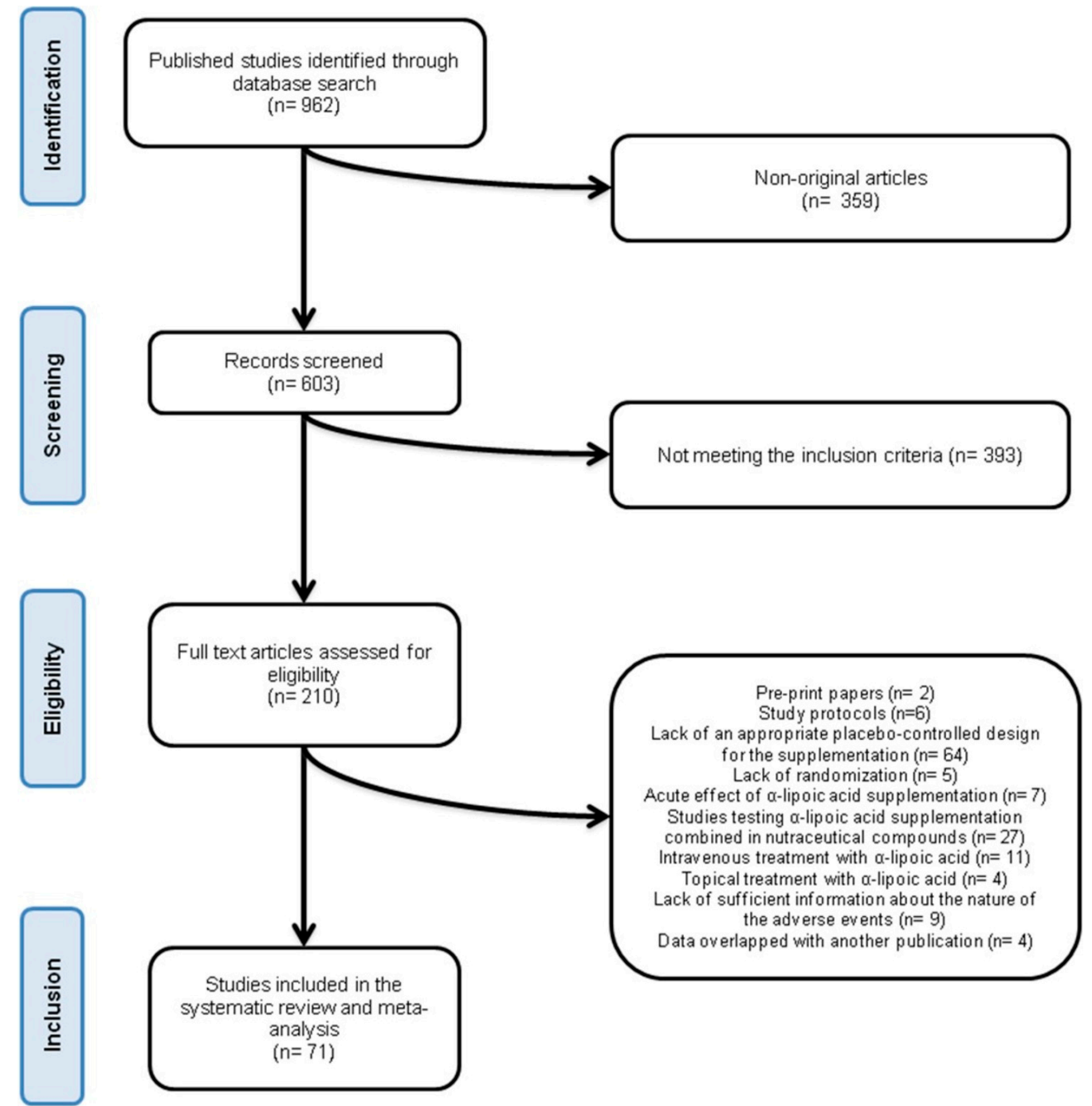

Figure 1. Flow chart of the number of studies identified and included in the systematic review.

Eligible studies were published between 1982 and 2020 and were conducted in different locations across all continents. Follow-up periods ranged between 8 days and 4 years and several ALA regimens were tested. Selected clinical trials were designed with cross-over or parallel-group and enrolled pregnant women with gestational diabetes, children and/or adolescent, overall healthy subjects or subjects with minor or major underlying diseases (e.g., diabetes, CVD, rheumatic affections, neurological disorders, severe renal impairment).

Included clinical studies were fully or partially carried out independently and funded by the National Institutes of Health $(n=7)$, Health Ministries $(n=2)$, University Institutes $(n=42)$, Research Hospitals $(n=2)$, Private Research Institutes $(n=2)$, Scientific Societies $(n=3)$, Private Foundations $(n=8)$, or were financially supported by industries $(n=7)$.

The main characteristics of the evaluated studies are summarized in Table 1. 
Table 1. Main characteristics of the clinical trials testing safety of treatment with $\alpha$-lipoic acid.

\begin{tabular}{|c|c|c|c|c|c|c|c|c|}
\hline Author, Year & Location & Study Design & $\begin{array}{l}\text { Treatment } \\
\text { Duration }\end{array}$ & $\begin{array}{l}\text { Main Inclusion Criteria and } \\
\text { Underlying Disease }\end{array}$ & Study Group & $\begin{array}{l}\text { Enrolled } \\
\text { Subjects } \\
(\mathbf{n})\end{array}$ & $\begin{array}{c}\text { Age } \\
\text { (years; } \\
\text { mean } \pm \text { SD) }\end{array}$ & $\begin{array}{c}\text { Male } \\
{[n(\%)]}\end{array}$ \\
\hline \multirow{2}{*}{$\begin{array}{l}\text { Ahmadi, } \\
2013[20]\end{array}$} & \multirow[t]{2}{*}{ Iran } & \multirow{2}{*}{$\begin{array}{l}\text { Randomized, single-blind, } \\
\text { placebo-controlled, parallel-group, } \\
\text { clinical study }\end{array}$} & \multirow[t]{2}{*}{2 months } & \multirow{2}{*}{$\begin{array}{l}\text { End-stage renal disease on haemodialysis } \\
\qquad(\geq 2 \text { times/week for } \geq 1 \text { year })\end{array}$} & $\begin{array}{l}600 \mathrm{mg} / \text { day } \\
\alpha \text {-lipoic acid }\end{array}$ & 20 & $48.8 \pm 11.2$ & $14(70)$ \\
\hline & & & & & Placebo & 24 & $48.9 \pm 12.5$ & $9(38)$ \\
\hline \multirow[t]{2}{*}{ Ansar, 2011 [21] } & \multirow[t]{2}{*}{ Iran } & \multirow{2}{*}{$\begin{array}{l}\text { Randomized, double-blind, } \\
\text { placebo-controlled, parallel-group, } \\
\text { clinical study }\end{array}$} & \multirow[t]{2}{*}{8 weeks } & \multirow{2}{*}{$\begin{array}{l}\text { Type } 2 \text { diabetes mellitus } \\
\text { FPG }>126 \mathrm{mg} / \mathrm{dL}\end{array}$} & $\begin{array}{l}300 \mathrm{mg} / \text { day } \\
\alpha \text {-lipoic acid }\end{array}$ & 29 & $49 \pm 9.1$ & $6(21)$ \\
\hline & & & & & Placebo & 28 & $51.8 \pm 8.3$ & $8(29)$ \\
\hline \multirow{2}{*}{$\begin{array}{l}\text { Aslfalah, } \\
\text { 2019a [22] }\end{array}$} & \multirow{2}{*}{ Iran } & \multirow{2}{*}{$\begin{array}{l}\text { Randomized, double-blind, } \\
\text { placebo-controlled, parallel-group, } \\
\text { clinical study }\end{array}$} & \multirow{2}{*}{8 weeks } & \multirow{2}{*}{ Gestational diabetes mellitus } & $\begin{array}{l}100 \mathrm{mg} / \text { day } \\
\alpha \text {-lipoic acid }\end{array}$ & 30 & $30.96 \pm 0.93$ & $0(0)$ \\
\hline & & & & & Placebo & 30 & $31.1 \pm 0.92$ & $0(0)$ \\
\hline \multirow{2}{*}{$\begin{array}{l}\text { Aslfalah, } \\
\text { 2019b [23] }\end{array}$} & \multirow{2}{*}{ Iran } & \multirow{2}{*}{$\begin{array}{l}\text { Randomized, double-blind, } \\
\text { placebo-controlled, parallel-group, } \\
\text { clinical study }\end{array}$} & \multirow{2}{*}{8 weeks } & \multirow{2}{*}{ Gestational diabetes mellitus } & $\begin{array}{l}100 \mathrm{mg} / \text { day } \\
\alpha \text {-lipoic acid }\end{array}$ & 30 & $30.96 \pm 0.93$ & $0(0)$ \\
\hline & & & & & Placebo & 30 & $31.1 \pm 0.92$ & $0(0)$ \\
\hline \multirow{2}{*}{$\begin{array}{l}\text { Baumgartner, } \\
2017[24]\end{array}$} & \multirow[t]{2}{*}{ The Netherlands } & \multirow{2}{*}{$\begin{array}{l}\text { Randomized, double-blind, } \\
\text { placebo-controlled, crossover, } \\
\text { clinical study }\end{array}$} & \multirow[t]{2}{*}{4 weeks } & \multirow{2}{*}{$\begin{array}{l}\text { Impaired glucose tolerance or } \\
\text { non-insulin-dependent type } 2 \text { diabetes } \\
\text { BMI } \geq 20 \mathrm{~kg} / \mathrm{m}^{2} \text { and } \leq 35 \mathrm{~kg} / \mathrm{m}^{2}\end{array}$} & $\begin{array}{l}600 \mathrm{mg} / \text { day } \\
\alpha \text {-lipoic acid }\end{array}$ & \multirow[t]{2}{*}{20} & \multirow[t]{2}{*}{$63.1 \pm 5.8$} & \multirow[t]{2}{*}{$16(80)$} \\
\hline & & & & & Placebo & & & \\
\hline \multirow[t]{2}{*}{ Baziar, 2020 [25] } & \multirow[t]{2}{*}{ Iran } & \multirow{2}{*}{$\begin{array}{l}\text { Randomized, double-blind, } \\
\text { placebo-controlled, parallel-group, } \\
\text { clinical study }\end{array}$} & \multirow[t]{2}{*}{8 weeks } & \multirow{2}{*}{$\begin{array}{l}\text { Non-insulin-dependent diabetes mellitus } \\
\text { HbA1c }<7 \% \\
\quad \mathrm{BMI} \geq 18.5 \mathrm{~kg} / \mathrm{m}^{2} \text { and } \leq 29.9 \mathrm{~kg} / \mathrm{m}^{2}\end{array}$} & $\begin{array}{l}1200 \mathrm{mg} / \text { day } \\
\alpha \text {-lipoic acid }\end{array}$ & 35 & $52.66 \pm 4.81$ & $15(43)$ \\
\hline & & & & & Placebo & 35 & $53.34 \pm 4.45$ & $16(46)$ \\
\hline \multirow[t]{2}{*}{ Bobe, 2020 [26] } & \multirow[t]{2}{*}{$\begin{array}{l}\text { United States of } \\
\text { America }\end{array}$} & \multirow[t]{2}{*}{$\begin{array}{l}\text { Randomized, double-blind, } \\
\text { placebo-controlled, parallel-group, } \\
\text { clinical study }\end{array}$} & 24 weeks & $\begin{array}{c}\text { Sedentary lifestyle } \\
\mathrm{BMI} \geq 27 \mathrm{~kg} / \mathrm{m}^{2} \\
\mathrm{TG} \geq 150 \mathrm{mg} / \mathrm{dL} \\
\mathrm{FPG}<125 \mathrm{mg} / \mathrm{dL}\end{array}$ & $\begin{array}{l}600 \mathrm{mg} / \text { day } \\
\alpha \text {-lipoic acid }\end{array}$ & 40 & $38 \pm 10 *$ & $12(39)$ * \\
\hline & & & & & Placebo & 41 & $40 \pm 8$ & $16(48)^{*}$ \\
\hline $\begin{array}{l}\text { Boriani, } \\
2017 \text { [27] }\end{array}$ & Italy & $\begin{array}{l}\text { Randomized, double-blind, } \\
\text { placebo-controlled, parallel-group, }\end{array}$ & 40 days & $\begin{array}{l}\text { Primary tunnel carpal syndrome } \\
\text { at least one of the following findings: } \\
\text { anaesthesia or paraesthesia in the median nerve }\end{array}$ & $\begin{array}{l}800 \mathrm{mg} / \text { day } \\
\alpha \text {-lipoic acid }\end{array}$ & 32 & $57.3 \pm 12$ & $13(41)$ \\
\hline & & & & & Placebo & 32 & $58.5+11$ & $9(28)$ \\
\hline
\end{tabular}


Table 1. Cont.

\begin{tabular}{|c|c|c|c|c|c|c|c|c|}
\hline Author, Year & Location & Study Design & $\begin{array}{l}\text { Treatment } \\
\text { Duration }\end{array}$ & $\begin{array}{l}\text { Main Inclusion Criteria and } \\
\text { Underlying Disease }\end{array}$ & Study Group & $\begin{array}{l}\text { Enrolled } \\
\text { Subjects } \\
\text { (n) }\end{array}$ & $\begin{array}{c}\text { Age } \\
\text { (years; } \\
\text { mean } \pm \text { SD) }\end{array}$ & $\begin{array}{l}\text { Male } \\
{[n(\%)]}\end{array}$ \\
\hline \multirow{2}{*}{$\begin{array}{l}\text { Carbone, } \\
2009[28]\end{array}$} & \multirow[t]{2}{*}{ Italy } & \multirow{2}{*}{$\begin{array}{l}\text { Randomized, double-blind, } \\
\text { placebo-controlled, parallel-group, } \\
\text { clinical study }\end{array}$} & \multirow[t]{2}{*}{8 weeks } & \multirow[t]{2}{*}{ Burning mouth syndrome } & $\begin{array}{l}800 \mathrm{mg} / \text { day } \\
\alpha \text {-lipoic acid }\end{array}$ & 22 & NA & NA \\
\hline & & & & & Placebo & 22 & NA & NA \\
\hline \multirow{2}{*}{$\begin{array}{l}\text { Cavalcanti, } \\
2009[29]\end{array}$} & \multirow{2}{*}{ Brazil } & \multirow{2}{*}{$\begin{array}{l}\text { Randomized, double-blind, } \\
\text { placebo-controlled, crossover, } \\
\text { clinical study }\end{array}$} & \multirow[t]{2}{*}{30 days } & \multirow[t]{2}{*}{ Burning mouth syndrome } & $\begin{array}{l}600 \mathrm{mg} / \text { day } \\
\alpha \text {-lipoic acid }\end{array}$ & \multirow[t]{2}{*}{38} & \multirow[t]{2}{*}{$63.1(36-78) \S$} & \multirow[t]{2}{*}{$4(11)$} \\
\hline & & & & & Placebo & & & \\
\hline \multirow[t]{2}{*}{$\begin{array}{l}\text { Durastanti, } \\
2016[30]\end{array}$} & \multirow[t]{2}{*}{ Italy } & \multirow[t]{2}{*}{$\begin{array}{l}\text { Randomized, double-blind, } \\
\text { placebo-controlled, parallel-group, } \\
\text { pilot clinical study }\end{array}$} & \multirow[t]{2}{*}{2 years } & \multirow[t]{2}{*}{$\begin{array}{l}\text { Relapsing-remitting multiple sclerosis } \\
\text { EDSS score } \leq 3.5\end{array}$} & $\begin{array}{l}800 \mathrm{mg} / \text { day } \\
\alpha \text {-lipoic acid during the } \\
\text { first year and } 400 \\
\mathrm{mg} / \text { day } \alpha \text {-lipoic acid } \\
\text { during the second year }\end{array}$ & 7 & $33(26-43)^{\circ}$ & $2(29)$ \\
\hline & & & & & Placebo & 6 & $\begin{array}{c}28.5 \\
(22.5-44.3)^{\circ}\end{array}$ & $1(17)$ \\
\hline \multirow{2}{*}{$\begin{array}{l}\text { El Amrousy, } \\
2020[31]\end{array}$} & \multirow[t]{2}{*}{ Egypt } & \multirow{2}{*}{$\begin{array}{l}\text { Randomized, double-blind, } \\
\text { placebo-controlled, parallel-group, } \\
\text { clinical study }\end{array}$} & \multirow[t]{2}{*}{3 months } & \multirow{2}{*}{$\begin{array}{l}\text { Obese healthy children and adolescents } \\
\text { BMI > 95th percentile for age and sex }\end{array}$} & $\begin{array}{l}600 \mathrm{mg} / \text { day } \\
\alpha \text {-lipoic acid }\end{array}$ & 40 & $12.3 \pm 1.5$ & $16(40)$ \\
\hline & & & & & Placebo & 40 & $12.4 \pm 1.4$ & $18(45)$ \\
\hline \multirow{2}{*}{$\begin{array}{l}\text { Falardeau, } \\
2019[32]\end{array}$} & \multirow{2}{*}{$\begin{array}{l}\text { United States of } \\
\text { America }\end{array}$} & \multirow{2}{*}{$\begin{array}{l}\text { Randomized, double-blind, } \\
\text { placebo-controlled, parallel-group, } \\
\text { clinical study }\end{array}$} & \multirow[t]{2}{*}{6 weeks } & \multirow[t]{2}{*}{ Unilateral acute optic neuritis } & $\begin{array}{l}1200 \mathrm{mg} / \mathrm{day} \\
\alpha \text {-lipoic acid }\end{array}$ & 15 & $41.2 \pm 10.51$ & $7(47)$ \\
\hline & & & & & Placebo & 16 & $36.1 \pm 9.84$ & $4(25)$ \\
\hline \multirow{2}{*}{$\begin{array}{l}\text { Femiano, } \\
2002[33]\end{array}$} & \multirow[t]{2}{*}{ Spain } & \multirow{2}{*}{$\begin{array}{l}\text { Randomized, double-blind, } \\
\text { placebo-controlled, parallel-group, } \\
\text { clinical study }\end{array}$} & \multirow{2}{*}{2 months } & \multirow[t]{2}{*}{ Burning mouth syndrome } & $\begin{array}{l}600 \mathrm{mg} / \text { day } \\
\alpha \text {-lipoic acid }\end{array}$ & 30 & \multirow[t]{2}{*}{$45(22-68)^{\S}$} & \multirow[t]{2}{*}{$18(30)$} \\
\hline & & & & & Placebo & 30 & & \\
\hline Georgakouli, & Greece & $\begin{array}{l}\text { Randomized, double-blind, } \\
\text { placebo-controlled, crossover, }\end{array}$ & 4 weeks & Healthy status & $\begin{array}{l}600 \mathrm{mg} / \text { day } \\
\alpha \text {-lipoic acid }\end{array}$ & 8 & $38.4 \pm 5.6$ & $8(100)$ \\
\hline & & clinical study & & & Placebo & & & \\
\hline Gianturco, & Italy & $\begin{array}{l}\text { Randomized, double-blind, } \\
\text { placebo-controlled, parallel-group, }\end{array}$ & 4 weeks & Diabetes mellitus & $\begin{array}{l}400 \mathrm{mg} / \text { day } \\
\alpha \text {-lipoic acid }\end{array}$ & 7 & $61 \pm 7$ & $4(57)$ \\
\hline 2009 [35] & & clinical study & & HbAlc $<7 \%$ & Placebo & 7 & $58 \pm 16$ & $4(57)$ \\
\hline Gilron, 2020 [36] & Canada & $\begin{array}{l}\text { Randomized, double-blind, } \\
\text { placebo-controlled, crossover, } \\
\text { clinical study }\end{array}$ & 5 weeks & $\begin{array}{c}\text { Fibromyalgia } \\
\text { daily moderate pain }(\geq 4 / 10 \text { on a NRS) for } \geq 3 \\
\text { months }\end{array}$ & $\begin{array}{c}600 \mathrm{mg} / \text { day } \\
\alpha \text {-lipoic acid during the } \\
\text { first week; } 1200 \mathrm{mg} / \text { day } \\
\alpha \text {-lipoic acid during the } \\
\text { second week; } 1800 \\
\text { mg/day } \alpha \text {-lipoic acid } \\
\text { during the third and } \\
\text { the fourth weeks }\end{array}$ & 27 & $57(25-74)^{\S}$ & $5(19)$ \\
\hline & & & & & Placebo & & & \\
\hline
\end{tabular}


Table 1. Cont.

\begin{tabular}{|c|c|c|c|c|c|c|c|c|}
\hline Author, Year & Location & Study Design & $\begin{array}{l}\text { Treatment } \\
\text { Duration }\end{array}$ & $\begin{array}{l}\text { Main Inclusion Criteria and } \\
\text { Underlying Disease }\end{array}$ & Study Group & $\begin{array}{l}\text { Enrolled } \\
\text { Subjects } \\
(\text { n) }\end{array}$ & $\begin{array}{c}\text { Age } \\
\text { (years; } \\
\text { mean } \pm \text { SD) }\end{array}$ & $\begin{array}{c}\text { Male } \\
{[n(\%)]}\end{array}$ \\
\hline \multirow{2}{*}{$\begin{array}{l}\text { Gosselin, } \\
2019[37]\end{array}$} & \multirow{2}{*}{$\begin{array}{l}\text { United States of } \\
\text { America }\end{array}$} & \multirow{2}{*}{$\begin{array}{l}\text { Randomized, double-blind, } \\
\text { placebo-controlled, crossover, } \\
\text { clinical study }\end{array}$} & \multirow{2}{*}{1 month } & \multirow{2}{*}{$\begin{array}{c}\text { Sedentary lifestyle } \\
\text { FPG } \geq 100 \mathrm{mg} / \mathrm{dL} \text { and } \leq 125 \mathrm{mg} / \mathrm{dL} \\
\mathrm{BMI} \geq 25 \mathrm{~kg} / \mathrm{m}^{2} \text { and } \leq 40 \mathrm{~kg} / \mathrm{m}^{2}\end{array}$} & $\begin{array}{l}600 \mathrm{mg} / \text { day } \\
\alpha \text {-lipoic acid }\end{array}$ & \multirow{2}{*}{12} & \multirow{2}{*}{$47.1 \pm 2.9$} & \multirow{2}{*}{$4(33)$} \\
\hline & & & & & Placebo & & & \\
\hline \multirow[t]{2}{*}{ Guo, 2014 [38] } & \multirow{2}{*}{$\begin{array}{l}\text { United States of } \\
\text { America }\end{array}$} & \multirow{2}{*}{$\begin{array}{l}\text { Randomized, double-blind, } \\
\text { placebo-controlled, parallel-group, } \\
\text { clinical study }\end{array}$} & \multirow[t]{2}{*}{24 weeks } & \multirow{2}{*}{$\begin{array}{l}\text { Cancer patients receiving chemotherapy with } \\
\text { cisplatin or oxaliplatin }\end{array}$} & $\begin{array}{l}1800 \mathrm{mg} / \mathrm{day} \\
\alpha \text {-lipoic acid }\end{array}$ & 122 & $55 \pm 11$ & $66(54)$ \\
\hline & & & & & Placebo & 121 & $57 \pm 12$ & $63(52)$ \\
\hline \multirow{2}{*}{$\begin{array}{l}\text { Haghighian, } \\
2015[39]\end{array}$} & \multirow[t]{2}{*}{ Iran } & \multirow{2}{*}{$\begin{array}{l}\text { Randomized, triple-blind, } \\
\text { placebo-controlled, parallel-group, } \\
\text { clinical study }\end{array}$} & \multirow[t]{2}{*}{12 weeks } & \multirow{2}{*}{$\begin{array}{l}\text { Idiopathic asthenozoospermia } \\
\mathrm{BMI}<30 \mathrm{~kg} / \mathrm{m}^{2}\end{array}$} & $\begin{array}{l}600 \mathrm{mg} / \text { day } \\
\alpha \text {-lipoic acid }\end{array}$ & 24 & $32.98 \pm 5.35$ * & $24(100)$ \\
\hline & & & & & Placebo & 24 & $34.12 \pm 4.79 *$ & $24(100)$ \\
\hline \multirow[t]{2}{*}{ Hejazi, 2018 [40] } & \multirow[t]{2}{*}{ Iran } & \multirow{2}{*}{$\begin{array}{l}\text { Randomized, double-blind, } \\
\text { placebo-controlled, parallel-group, } \\
\text { clinical study }\end{array}$} & \multirow[t]{2}{*}{10 days } & \multirow{2}{*}{$\begin{array}{l}\text { Candidates for enteral feeding and expected to } \\
\text { stay in the intensive care unit for } \geq 7 \text { days }\end{array}$} & $\begin{array}{l}2700 \mathrm{mg} / \text { day } \\
\alpha \text {-lipoic acid }\end{array}$ & 40 & $51.2 \pm 17$ & $17(43)$ \\
\hline & & & & & Placebo & 40 & $57.4 \pm 19$ & $25(63)$ \\
\hline \multirow[t]{2}{*}{$\begin{array}{l}\text { Huang, } \\
2008[41]\end{array}$} & \multirow[t]{2}{*}{$\begin{array}{l}\text { United States of } \\
\text { America }\end{array}$} & \multirow{2}{*}{$\begin{array}{c}\text { Randomized, double-blind, } \\
\text { placebo-controlled, parallel-group, } \\
\text { clinical study }\end{array}$} & \multirow[t]{2}{*}{3 months } & \multirow[t]{2}{*}{$\begin{array}{l}\text { Pubertal or postpubertal adolescents with type } \\
1 \text { diabetes }\end{array}$} & $\begin{array}{c}600-1200 \mathrm{mg} / \text { day } \\
(14-21 \mathrm{mg} / \mathrm{kg} / \text { day }) \\
\alpha \text {-lipoic acid }\end{array}$ & 30 & $14 \pm 2.4$ & $13(43)$ \\
\hline & & & & & Placebo & 10 & $15 \pm 1.9$ & $7(70)$ \\
\hline \multirow{2}{*}{$\begin{array}{l}\text { Huerta, } \\
2016[42]\end{array}$} & \multirow[t]{2}{*}{ Spain } & \multirow{2}{*}{$\begin{array}{l}\text { Randomized, double-blind, } \\
\text { placebo-controlled, parallel-group, } \\
\text { clinical study }\end{array}$} & \multirow[t]{2}{*}{10 weeks } & \multirow{2}{*}{$\begin{array}{c}\text { Sedentary lifestyle } \\
\mathrm{BMI} \geq 27.5 \mathrm{~kg} / \mathrm{m}^{2} \text { and } \leq 40 \mathrm{~kg} / \mathrm{m}^{2}\end{array}$} & $\begin{array}{l}300 \mathrm{mg} / \text { day } \\
\alpha \text {-lipoic acid }\end{array}$ & 6 & $35.5 \pm 8.4$ & $0(0)$ \\
\hline & & & & & Placebo & 6 & $41.8 \pm 6.6$ & $0(0)$ \\
\hline Huerta, & Spain & $\begin{array}{l}\text { Randomized, double-blind, } \\
\text { placebo-controlled, parallel-group, }\end{array}$ & 10 weeks & $\begin{array}{l}\text { Healthy status } \\
\text { regular menstrual cycles }\end{array}$ & $\begin{array}{l}300 \mathrm{mg} / \text { day } \\
\alpha \text {-lipoic acid }\end{array}$ & 26 & $39 \pm 8^{*}$ & $0(0)$ \\
\hline & & clinical study & & BMI $\geq 27.5 \mathrm{~kg} / \mathrm{m}^{2}$ and $\leq 40 \mathrm{~kg} / \mathrm{m}^{2}$ & Placebo & 31 & $38 \pm 7^{*}$ & $0(0)$ \\
\hline & & & & & $\begin{array}{l}1800 \mathrm{mg} / \text { day } \\
\alpha \text {-lipoic acid }\end{array}$ & 18 & $62.1 \pm 3$ & $10(56)$ \\
\hline Jacob, 1999 [44] & Germany & $\begin{array}{l}\text { Randomized, double-blind, } \\
\text { placebo-controlled, parallel-group, }\end{array}$ & 4 weeks & Well-controlled type 2 diabetes mellitus & $\begin{array}{l}1200 \mathrm{mg} / \mathrm{day} \\
\alpha \text {-lipoic acid }\end{array}$ & 18 & $60.9 \pm 2.2$ & $11(61)$ \\
\hline & & & & & $\begin{array}{l}600 \mathrm{mg} / \text { day } \\
\alpha \text {-lipoic acid }\end{array}$ & 19 & $58.1 \pm 2.8$ & $10(53)$ \\
\hline & & & & & Placebo & 19 & $60.4 \pm 2.4$ & $12(63)$ \\
\hline Jamshidi, & Iran & $\begin{array}{l}\text { Randomized, double-blind, } \\
\text { Rando-controlled }\end{array}$ & 8 weeks & $\beta$-thalassemia major & $\begin{array}{l}600 \mathrm{mg} / \text { day } \\
\alpha \text {-lipoic acid }\end{array}$ & 20 & $23.5+5.47$ & $13(65)$ \\
\hline & & clinical study & & & Placebo & & & \\
\hline $\begin{array}{l}\text { Jariwalla, } \\
2008[46]\end{array}$ & $\begin{array}{l}\text { United States of } \\
\text { America }\end{array}$ & $\begin{array}{l}\text { Randomized, double-blind, } \\
\text { placebo-controlled, parallel-group, }\end{array}$ & 6 months & $\begin{array}{l}\text { HIV infection } \\
\text { HIV-RNA viral load }>10.000 \text { copies } / \mathrm{cm}^{3} \\
\text { despite HAART }\end{array}$ & $\begin{array}{l}900 \mathrm{mg} / \text { day } \\
\alpha \text {-lipoic acid }\end{array}$ & 18 & $47.2 \pm 6.8$ & $29(88)$ \\
\hline & & & & CD $4+$ cell count $\geq 50$ cells $/ \mathrm{mm}^{3}$ & Placebo & 15 & $43.7 \pm 7.6$ & \\
\hline
\end{tabular}


Table 1. Cont.

\begin{tabular}{|c|c|c|c|c|c|c|c|c|}
\hline Author, Year & Location & Study Design & $\begin{array}{l}\text { Treatment } \\
\text { Duration }\end{array}$ & $\begin{array}{l}\text { Main Inclusion Criteria and } \\
\text { Underlying Disease }\end{array}$ & Study Group & $\begin{array}{l}\text { Enrolled } \\
\text { Subjects } \\
(\mathbf{n})\end{array}$ & $\begin{array}{c}\text { Age } \\
\text { (years; } \\
\text { mean } \pm \text { SD) }\end{array}$ & $\begin{array}{c}\text { Male } \\
{[n(\%)]}\end{array}$ \\
\hline \multirow{2}{*}{$\begin{array}{l}\text { Khabbazi, } \\
2012[47]\end{array}$} & \multirow{2}{*}{ Iran } & \multirow{2}{*}{$\begin{array}{l}\text { Randomized, double-blind, } \\
\text { placebo-controlled, parallel-group, } \\
\text { clinical study }\end{array}$} & \multirow{2}{*}{8 weeks } & \multirow{2}{*}{$\begin{array}{l}\text { Patients with end-stage renal disease on } \\
\text { haemodialysis }\end{array}$} & $\begin{array}{l}600 \mathrm{mg} / \text { day } \\
\alpha \text {-lipoic acid }\end{array}$ & 31 & $53.83 \pm 13.29$ & $16(52)$ \\
\hline & & & & & Placebo & 32 & $54.04 \pm 13.96$ & $18(56)$ \\
\hline \multirow{2}{*}{$\begin{array}{l}\text { Khalili, } \\
2017[48]\end{array}$} & \multirow[t]{2}{*}{ Iran } & \multirow{2}{*}{$\begin{array}{l}\text { Randomized, double-blind, } \\
\text { placebo-controlled, parallel-group, } \\
\text { clinical study }\end{array}$} & \multirow{2}{*}{12 weeks } & \multirow[t]{2}{*}{ Relapsing-remitting multiple sclerosis } & $\begin{array}{l}1200 \mathrm{mg} / \mathrm{day} \\
\alpha \text {-lipoic acid }\end{array}$ & 15 & $32.3 \pm 6.2 *$ & $5(42) *$ \\
\hline & & & & & Placebo & 16 & $32.2 \pm 10.5^{*}$ & $1(8)^{*}$ \\
\hline \multirow{2}{*}{$\begin{array}{l}\text { Khalili, } \\
2014[49]\end{array}$} & \multirow{2}{*}{ Iran } & \multirow{2}{*}{$\begin{array}{l}\text { Randomized, double-blind, } \\
\text { placebo-controlled, parallel-group, } \\
\text { clinical study }\end{array}$} & \multirow{2}{*}{12 weeks } & \multirow{2}{*}{ Relapsing-remitting multiple sclerosis } & $\begin{array}{l}1200 \mathrm{mg} / \text { day } \\
\alpha \text {-lipoic acid }\end{array}$ & 26 & $31.4 \pm 6.2 *$ & $7(27)$ \\
\hline & & & & & Placebo & 34 & $28.7 \pm 9 *$ & $9(26)$ \\
\hline \multirow[t]{2}{*}{ Kim, 2020 [50] } & \multirow[t]{2}{*}{ South Korea } & \multirow{2}{*}{$\begin{array}{l}\text { Randomized, double-blind, } \\
\text { placebo-controlled, parallel-group, } \\
\text { clinical study }\end{array}$} & \multirow[t]{2}{*}{18 months } & \multirow[t]{2}{*}{ Geographic atrophy } & $\begin{array}{l}1200 \mathrm{mg} / \text { day } \\
\alpha \text {-lipoic acid }\end{array}$ & 26 & $80.6 \pm 6.5$ & $8(31)$ \\
\hline & & & & & Placebo & 27 & $79 \pm 7$ & $11(41)$ \\
\hline \multirow[t]{2}{*}{ Kim, 2016 [51] } & \multirow[t]{2}{*}{ South Korea } & \multirow{2}{*}{$\begin{array}{l}\text { Randomized, double-blind, } \\
\text { placebo-controlled, parallel-group, } \\
\text { clinical study }\end{array}$} & \multirow[t]{2}{*}{12 weeks } & \multirow{2}{*}{$\begin{array}{l}\text { Chronic schizophrenia in rehabilitation } \\
\text { significant weight gain after starting treatment } \\
\text { with atypical antipsychotics }\end{array}$} & $\begin{array}{c}600-1800 \mathrm{mg} / \text { day } \\
\alpha \text {-lipoic acid }\end{array}$ & 10 & $40.5 \pm 6.65$ & $4(40)$ \\
\hline & & & & & Placebo & 12 & $40.08 \pm 9.14$ & $7(58)$ \\
\hline \multirow{3}{*}{ Koh, 2011 [52] } & \multirow{3}{*}{$\begin{array}{l}\text { Republic of } \\
\text { Korea }\end{array}$} & \multirow{3}{*}{$\begin{array}{l}\text { Randomized, double-blind, } \\
\text { placebo-controlled, parallel-group, } \\
\text { clinical study }\end{array}$} & \multirow{3}{*}{20 weeks } & \multirow{3}{*}{$\begin{array}{c}\mathrm{BMI} \geq 30 \mathrm{~kg} / \mathrm{m}^{2} \text { or } \mathrm{BMI} \geq 27.5 \mathrm{~kg} / \mathrm{m}^{2} \text { and } \leq 40 \\
\mathrm{~kg} / \mathrm{m}^{2} \text { if hypertension, diabetes mellitus and } / \text { or } \\
\text { hypercholesterolemia coexisted }\end{array}$} & $\begin{array}{l}1800 \mathrm{mg} / \text { day } \\
\alpha \text {-lipoic acid }\end{array}$ & 120 & $41.4 \pm 1$ & $82(68)$ \\
\hline & & & & & $\begin{array}{l}1200 \mathrm{mg} / \text { day } \\
\alpha \text {-lipoic acid }\end{array}$ & 120 & $41.6 \pm 1.1$ & $79(66)$ \\
\hline & & & & & Placebo & 120 & $40.7 \pm 1.1$ & $74(62)$ \\
\hline \multirow[t]{2}{*}{$\begin{array}{l}\text { Lampitella, } \\
2005 \text { [53] }\end{array}$} & \multirow[t]{2}{*}{ Italy } & \multirow{2}{*}{$\begin{array}{l}\text { Randomized, double-blind, } \\
\text { placebo-controlled, parallel-group, } \\
\text { clinical study }\end{array}$} & \multirow[t]{2}{*}{6 months } & Type 2 diabetes mellitus & $\begin{array}{l}600 \mathrm{mg} / \text { day } \\
\alpha \text {-lipoic acid }\end{array}$ & 20 & NA & NA \\
\hline & & & & & Placebo & 20 & NA & NA \\
\hline Lee, 2017 [54] & Republic of & $\begin{array}{l}\text { Randomized, double-blind, } \\
\text { placebo-controlled, parallel-group, }\end{array}$ & 24 weeks & Diabetic cardiac autonomic neuropathy & $\begin{array}{c}600-1200 \mathrm{mg} / \text { day } \\
\alpha \text {-lipoic acid }\end{array}$ & 46 & $64.37 \pm 7.8$ & $27(59)$ \\
\hline & & clinical study & & & Placebo & 45 & $62.4 \pm 9.1$ & $20(44)$ \\
\hline Loy, 2018 [55] & $\begin{array}{l}\text { United States of } \\
\text { America }\end{array}$ & $\begin{array}{l}\text { Randomized, double-blind, } \\
\text { placebo-controlled, parallel-group, } \\
\text { pilot clinical study }\end{array}$ & 2 years & $\begin{array}{l}\text { Multiple sclerosis disability progression in } \\
\text { absence of clinical relapse for } 5 \text { years } \\
\text { EDSS } \leq 6.0 \\
\text { ability to walk } \geq 25 \text { feet without aid }\end{array}$ & $\begin{array}{l}1200 \mathrm{mg} / \text { day } \\
\alpha \text {-lipoic acid }\end{array}$ & 11 & $55.8 \pm 5.7$ & $5(45)$ \\
\hline & & & & & Placebo & 10 & $55.7 \pm 4.1$ & $5(50)$ \\
\hline López-D'alessandro, & Argentina & $\begin{array}{l}\text { Randomized, double-blind, } \\
\text { placebo-controlled }\end{array}$ & 2 months & Burning mouth syndrome & $600 \mathrm{~g} /$ day $\alpha$-lipoic acid & 20 & NA & NA \\
\hline $2011[56]$ & Argentina & $\begin{array}{l}\text { placebo-controlled, parallel-group, } \\
\text { clinical study }\end{array}$ & 2 montns & & Placebo & 60 & NA & NA \\
\hline
\end{tabular}


Table 1. Cont.

\begin{tabular}{|c|c|c|c|c|c|c|c|c|}
\hline Author, Year & Location & Study Design & $\begin{array}{l}\text { Treatment } \\
\text { Duration }\end{array}$ & $\begin{array}{l}\text { Main Inclusion Criteria and } \\
\text { Underlying Disease }\end{array}$ & Study Group & $\begin{array}{l}\text { Enrolled } \\
\text { Subjects } \\
\text { (n) }\end{array}$ & $\begin{array}{c}\text { Age } \\
\text { (years; } \\
\text { mean } \pm \text { SD) }\end{array}$ & $\begin{array}{c}\text { Male } \\
{[n(\%)]}\end{array}$ \\
\hline \multirow{2}{*}{$\begin{array}{l}\text { López-Jornet, } \\
2009 \text { [57] }\end{array}$} & \multirow[t]{2}{*}{ Spain } & \multirow{2}{*}{$\begin{array}{l}\text { Randomized, double-blind, } \\
\text { placebo-controlled, parallel-group, } \\
\text { clinical study }\end{array}$} & \multirow[t]{2}{*}{8 weeks } & \multirow[t]{2}{*}{ Burning mouth syndrome } & $\begin{array}{l}800 \mathrm{mg} / \text { day } \\
\alpha \text {-lipoic acid }\end{array}$ & 30 & \multirow[t]{2}{*}{$64.37 \pm 11.61$} & \multirow[t]{2}{*}{$6(10)$} \\
\hline & & & & & Placebo & 30 & & \\
\hline \multirow[t]{2}{*}{ Magis, 2007 [58] } & \multirow[t]{2}{*}{ Belgium } & \multirow{2}{*}{$\begin{array}{l}\text { Randomized, double-blind, } \\
\text { placebo-controlled, parallel-group, } \\
\text { clinical study }\end{array}$} & \multirow[t]{2}{*}{3 months } & \multirow[t]{2}{*}{ Migraine with or without aura } & $\begin{array}{l}600 \mathrm{mg} / \text { day } \\
\alpha \text {-lipoic acid }\end{array}$ & 26 & $37.46 \pm 13.43$ & $4(15)$ \\
\hline & & & & & Placebo & 18 & $38.94 \pm 8.05$ & $2(11)$ \\
\hline \multirow{2}{*}{$\begin{array}{l}\text { Manning, } \\
2013 \text { [59] }\end{array}$} & \multirow{2}{*}{ New Zeland } & \multirow{2}{*}{$\begin{array}{l}\text { Randomized, double-blind, } \\
\text { placebo-controlled, parallel-group, } \\
\text { clinical study }\end{array}$} & \multirow[t]{2}{*}{1 year } & \multirow[t]{2}{*}{ Metabolic syndrome } & $\begin{array}{l}600 \mathrm{mg} / \text { day } \\
\alpha \text {-lipoic acid }\end{array}$ & 34 & $55 \pm 10$ & $14(41)$ \\
\hline & & & & & Placebo & 40 & $57 \pm 9$ & $15(38)$ \\
\hline \multirow{2}{*}{$\begin{array}{l}\text { Marfella, } \\
2016[60]\end{array}$} & \multirow[t]{2}{*}{ Italy } & \multirow{2}{*}{$\begin{array}{l}\text { Randomized, double-blind, } \\
\text { placebo-controlled, parallel-group, } \\
\text { clinical study }\end{array}$} & \multirow{2}{*}{12 months } & \multirow[t]{2}{*}{ Takotsubo cadiomyopathy } & $\begin{array}{l}600 \mathrm{mg} / \text { day } \\
\alpha \text {-lipoic acid }\end{array}$ & 24 & $63.7 \pm 6.5$ & $0(0)$ \\
\hline & & & & & Placebo & 24 & $63.9 \pm 5.2$ & $0(0)$ \\
\hline \multirow{2}{*}{$\begin{array}{l}\text { Marshall, } \\
1982[61]\end{array}$} & \multirow{2}{*}{$\begin{array}{l}\text { United } \\
\text { Kingdom }\end{array}$} & \multirow{2}{*}{$\begin{array}{l}\text { Randomized, double-blind, } \\
\text { placebo-controlled, parallel-group, } \\
\text { clinical study }\end{array}$} & \multirow{2}{*}{24 weeks } & \multirow{2}{*}{ Alcohol related liver disease } & $\begin{array}{l}300 \mathrm{mg} / \text { day } \\
\alpha \text {-lipoic acid }\end{array}$ & 20 & $50.7 \pm 1.9$ & $17(85)$ \\
\hline & & & & & Placebo & 20 & $46.4 \pm 2.7$ & $15(75)$ \\
\hline \multirow{6}{*}{$\begin{array}{l}\text { Martins, } \\
2009[62]\end{array}$} & \multirow{6}{*}{ Brazil } & \multirow{6}{*}{$\begin{array}{l}\text { Randomized, double-blind, } \\
\text { placebo-controlled, parallel-group, } \\
\text { clinical study }\end{array}$} & \multirow{6}{*}{3 months } & \multirow[t]{2}{*}{ Sickle cell disease } & $\begin{array}{l}200 \mathrm{mg} / \text { day } \\
\alpha \text {-lipoic acid }\end{array}$ & 10 & $17.7 \pm 9.6$ & $6(60)$ \\
\hline & & & & & Placebo & 10 & $17 \pm 11$ & $5(50)$ \\
\hline & & & & \multirow[t]{2}{*}{ Sickle cell trait } & $\begin{array}{l}200 \mathrm{mg} / \text { day } \\
\alpha \text {-lipoic acid }\end{array}$ & 10 & $31.3 \pm 15.4$ & $2(20)$ \\
\hline & & & & & Placebo & 10 & $29.7 \pm 10.8$ & $2(20)$ \\
\hline & & & & \multirow[t]{2}{*}{ Healthy status } & $\begin{array}{l}200 \mathrm{mg} / \text { day } \\
\alpha \text {-lipoic acid }\end{array}$ & 10 & $23.5 \pm 11$ & $4(40)$ \\
\hline & & & & & Placebo & 10 & $23.3 \pm 11$ & $3(30)$ \\
\hline $\begin{array}{l}\text { Mendes, } \\
2014[63]\end{array}$ & Brazil & $\begin{array}{l}\text { Randomized, double-blind, } \\
\text { placebo-controlled, parallel-group, }\end{array}$ & 12 weeks & Arterial hypertension & $\begin{array}{l}600 \mathrm{mg} / \text { day } \\
\alpha \text {-lipoic acid }\end{array}$ & 32 & NA & NA \\
\hline & & clinical study & & & Placebo & 28 & NA & NA \\
\hline $\begin{array}{l}\text { Mendoza-Núñez, } \\
2019 \text { [64] }\end{array}$ & Mexico & $\begin{array}{l}\text { Randomized, double-blind, } \\
\text { placebo-controlled, parallel-group, }\end{array}$ & 6 months & $\begin{array}{l}\text { Type } 2 \text { diabetes mellitus without complications } \\
\text { or comorbidity, treated with two tablets of } \\
\text { glibenclamide } / \text { metformin }(5 / 500 \mathrm{mg} \text { ) per day }\end{array}$ & $\begin{array}{l}600 \mathrm{mg} / \text { day } \\
\alpha \text {-lipoic acid }\end{array}$ & 50 & $63 \pm 1 *$ & NA \\
\hline & & & & sedentary lifestyle & Placebo & 50 & $64 \pm 1 *$ & NA \\
\hline
\end{tabular}


Table 1. Cont

\begin{tabular}{|c|c|c|c|c|c|c|c|c|}
\hline Author, Year & Location & Study Design & $\begin{array}{l}\text { Treatment } \\
\text { Duration }\end{array}$ & $\begin{array}{l}\text { Main Inclusion Criteria and } \\
\text { Underlying Disease }\end{array}$ & Study Group & $\begin{array}{l}\text { Enrolled } \\
\text { Subjects } \\
\text { (n) }\end{array}$ & $\begin{array}{c}\text { Age } \\
\text { (years; } \\
\text { mean } \pm \text { SD) }\end{array}$ & $\begin{array}{c}\text { Male } \\
{[n(\%)]}\end{array}$ \\
\hline \multirow{2}{*}{$\begin{array}{l}\text { Mirtaheri, } \\
2014 \text { [65] }\end{array}$} & \multirow{2}{*}{ Iran } & \multirow{2}{*}{$\begin{array}{l}\text { Randomized, double-blind, } \\
\text { placebo-controlled, parallel-group, } \\
\text { clinical study }\end{array}$} & \multirow{2}{*}{8 weeks } & \multirow{2}{*}{ Rheumatoid arthritis } & $\begin{array}{l}1200 \mathrm{mg} / \text { day } \\
\alpha \text {-lipoic acid }\end{array}$ & 35 & $36.09 \pm 8.77^{*}$ & $0(0)$ \\
\hline & & & & & Placebo & 35 & $38.28 \pm 8.63 *$ & $0(0)$ \\
\hline \multirow{2}{*}{$\begin{array}{l}\text { Mohammadi, } \\
2018[66]\end{array}$} & \multirow{2}{*}{ Iran } & \multirow{2}{*}{$\begin{array}{l}\text { Randomized, double-blind, } \\
\text { placebo-controlled, parallel-group, } \\
\text { clinical study }\end{array}$} & \multirow{2}{*}{12 weeks } & \multirow{2}{*}{$\begin{array}{l}\text { Previous thrombotic or embolic stroke } \\
\quad \mathrm{BMI} \geq 18.5 \mathrm{~kg} / \mathrm{m}^{2} \text { and } \leq 35 \mathrm{~kg} / \mathrm{m}^{2}\end{array}$} & $\begin{array}{l}600 \mathrm{mg} / \text { day } \\
\alpha \text {-lipoic acid }\end{array}$ & 40 & $62.33 \pm 6.19$ & NA \\
\hline & & & & & Placebo & 40 & $64.23 \pm 8.01$ & NA \\
\hline \multirow{2}{*}{$\begin{array}{l}\text { Mohammadi, } \\
2015[67]\end{array}$} & \multirow{2}{*}{ Iran } & \multirow{2}{*}{$\begin{array}{l}\text { Randomized, double-blind, } \\
\text { placebo-controlled, parallel-group, } \\
\text { clinical study }\end{array}$} & \multirow{2}{*}{12 weeks } & \multirow{2}{*}{$\begin{array}{l}\text { Spinal cord injury since } \geq 1 \text { year } \\
\text { BMI } \geq 18.5 \mathrm{~kg} / \mathrm{m}^{2}\end{array}$} & $\begin{array}{l}600 \mathrm{mg} / \text { day } \\
\alpha \text {-lipoic acid }\end{array}$ & 28 & $39 \pm 6.44$ & $28(100)$ \\
\hline & & & & & Placebo & 30 & $36.8 \pm 7.48$ & $30(100)$ \\
\hline \multirow[t]{2}{*}{ Mollo, 2012 [68] } & \multirow[t]{2}{*}{ Italy } & \multirow{2}{*}{$\begin{array}{l}\text { Randomized, double-blind, } \\
\text { placebo-controlled, parallel-group, } \\
\text { clinical study }\end{array}$} & \multirow[t]{2}{*}{5 weeks } & \multirow[t]{2}{*}{ Type 1 diabetes } & $\begin{array}{l}600 \mathrm{mg} / \text { day } \\
\alpha \text {-lipoic acid }\end{array}$ & 26 & $43 \pm 9$ & $15(58)$ \\
\hline & & & & & Placebo & 25 & $46 \pm 11$ & $12(48)$ \\
\hline \multirow{2}{*}{$\begin{array}{l}\text { Monroy Guízar, } \\
2018 \text { [69] }\end{array}$} & \multirow[t]{2}{*}{ Mexico } & \multirow{2}{*}{$\begin{array}{l}\text { Randomized, double-blind, } \\
\text { placebo-controlled, parallel-group, } \\
\text { clinical study }\end{array}$} & \multirow[t]{2}{*}{3 months } & \multirow[t]{2}{*}{ Idiopathic carpal tunnel syndrome } & $\begin{array}{l}600 \mathrm{mg} / \text { day } \\
\alpha \text {-lipoic acid }\end{array}$ & 10 & $45.3^{+}$ & $1(10)$ \\
\hline & & & & & Placebo & 10 & $48.4^{+}$ & $1(10)$ \\
\hline \multirow{2}{*}{$\begin{array}{l}\text { Palacios-Sánchez, } \\
2015 \text { [70] }\end{array}$} & \multirow[t]{2}{*}{ Spain } & \multirow{2}{*}{$\begin{array}{c}\text { Randomized, double-blind, } \\
\text { placebo-controlled, parallel-group, } \\
\text { clinical study }\end{array}$} & \multirow[t]{2}{*}{2 months } & \multirow[t]{2}{*}{ Burning mouth syndrome } & $\begin{array}{l}600 \mathrm{mg} / \text { day } \\
\alpha \text {-lipoic acid }\end{array}$ & 30 & \multirow[t]{2}{*}{$62.13(36-86)^{\S}$} & \multirow[t]{2}{*}{$5(8)$} \\
\hline & & & & & Placebo & 30 & & \\
\hline \multirow{5}{*}{$\begin{array}{l}\text { Porasuphatana, } \\
\quad 2012[71]\end{array}$} & \multirow{5}{*}{ Thailand } & & & & $\begin{array}{l}1200 \mathrm{mg} / \mathrm{day} \\
\alpha \text {-lipoic acid }\end{array}$ & 7 & $47.07 \pm 2.18$ & $1(14)$ \\
\hline & & Randomized, double-blind, & & & $\begin{array}{l}900 \mathrm{mg} / \text { day } \\
\alpha \text {-lipoic acid }\end{array}$ & 7 & $44 \pm 2$ & $1(14)$ \\
\hline & & $\begin{array}{l}\text { placebo-controlled, parallel-group, } \\
\text { clinical study }\end{array}$ & 6 months & Type 2 diabetes mellitus with microalbuminuria & $\begin{array}{l}600 \mathrm{mg} / \text { day } \\
\alpha \text {-lipoic acid }\end{array}$ & 8 & $45.7 \pm 1.68$ & $3(38)$ \\
\hline & & & & & $\begin{array}{l}300 \mathrm{mg} / \text { day } \\
\alpha \text {-lipoic acid }\end{array}$ & 8 & $42.5 \pm 1.12$ & $4(50)$ \\
\hline & & & & & Placebo & 8 & $42.9 \pm 2.52$ & $1(13)$ \\
\hline $\begin{array}{l}\text { Pourghasem } \\
\text { Gargari, }\end{array}$ & Iran & $\begin{array}{l}\text { Randomized, double-blind, } \\
\text { placebo-controlled, parallel-group, }\end{array}$ & 8 weeks & $\begin{array}{l}\text { Rheumatoid arthritis } \\
\text { DAS28 }<5.1\end{array}$ & $\begin{array}{l}1200 \mathrm{mg} / \text { day } \\
\alpha \text {-lipoic acid }\end{array}$ & 35 & $36.1 \pm 8.8$ & $0(0)$ \\
\hline 2014 [72] & & clinical study & & $\mathrm{BMI}<40 \mathrm{~kg} / \mathrm{m}^{2}$ & Placebo & 35 & $38.3 \pm 8.6$ & $0(0)$ \\
\hline Rahmanabadi, & Iran & $\begin{array}{l}\text { Randomized, double-blind, } \\
\text { placebo-controlled, parallel-group, }\end{array}$ & 12 weeks & Non-alcoholic fatty liver disease & $\begin{array}{l}1200 \mathrm{mg} / \mathrm{day} \\
\alpha \text {-lipoic acid }\end{array}$ & 25 & $40.28 \pm 5.5$ & $13(52)$ \\
\hline & & clinical study & & & Placebo & 25 & $37.52 \pm 9.67$ & $14(56)$ \\
\hline
\end{tabular}


Table 1. Cont.

\begin{tabular}{|c|c|c|c|c|c|c|c|c|}
\hline Author, Year & Location & Study Design & $\begin{array}{l}\text { Treatment } \\
\text { Duration }\end{array}$ & $\begin{array}{l}\text { Main Inclusion Criteria and } \\
\text { Underlying Disease }\end{array}$ & Study Group & $\begin{array}{l}\text { Enrolled } \\
\text { Subjects } \\
\text { (n) }\end{array}$ & $\begin{array}{c}\text { Age } \\
\text { (years; } \\
\text { mean } \pm \text { SD) }\end{array}$ & $\begin{array}{c}\text { Male } \\
{[n(\%)]}\end{array}$ \\
\hline \multirow{2}{*}{$\begin{array}{l}\text { Ruhnau, } \\
1999[73]\end{array}$} & \multirow[t]{2}{*}{ Germany } & \multirow{2}{*}{$\begin{array}{l}\text { Randomized, double-blind, } \\
\text { placebo-controlled, parallel-group, } \\
\text { clinical study }\end{array}$} & \multirow[t]{2}{*}{3 weeks } & \multirow{2}{*}{$\begin{array}{c}\text { Type } 2 \text { diabetes mellitus with distal symmetrical } \\
\text { polyneuropathy }\end{array}$} & $\begin{array}{l}1800 \mathrm{mg} / \mathrm{day} \\
\alpha \text {-lipoic acid }\end{array}$ & 12 & $60.5 \pm 6.9$ & $6(50)$ \\
\hline & & & & & Placebo & 12 & $62.1 \pm 4.5$ & $6(50)$ \\
\hline \multirow[t]{2}{*}{ Safa, 2014 [74] } & \multirow[t]{2}{*}{ Iran } & \multirow{2}{*}{$\begin{array}{l}\text { Randomized, double-blind, } \\
\text { placebo-controlled, parallel-group, } \\
\text { clinical study }\end{array}$} & \multirow[t]{2}{*}{12 months } & \multirow{2}{*}{$\begin{array}{c}\text { End-stage renal disease on haemodialysis } \geq 6 \\
\text { months }\end{array}$} & $\begin{array}{l}600 \mathrm{mg} / \text { day } \\
\alpha \text {-lipoic acid }\end{array}$ & 30 & $59.3 \pm 10.47$ & $21(70)$ \\
\hline & & & & & Placebo & 31 & $55.2 \pm 13.43$ & $21(68)$ \\
\hline \multirow{2}{*}{$\begin{array}{l}\text { Sammour, } \\
2019[75]\end{array}$} & \multirow[t]{2}{*}{ Egypt } & \multirow{2}{*}{$\begin{array}{l}\text { Randomized, triple-blind, } \\
\text { placebo-controlled, parallel-group, } \\
\text { clinical study }\end{array}$} & \multirow[t]{2}{*}{6 weeks } & \multirow{2}{*}{$\begin{array}{c}\text { Primary caesarean section in singleton term } \\
\text { pregnancy }\end{array}$} & $\begin{array}{l}1200 \mathrm{mg} / \text { day } \\
\alpha \text {-lipoic acid }\end{array}$ & 51 & $25.3 \pm 5.1$ & $0(0)$ \\
\hline & & & & & Placebo & 51 & $25.1 \pm 5.4$ & $0(0)$ \\
\hline \multirow[t]{2}{*}{ Sardu, 2017 [76] } & \multirow[t]{2}{*}{ Italy } & \multirow{2}{*}{$\begin{array}{l}\text { Randomized, double-blind, } \\
\text { placebo-controlled, parallel-group, } \\
\text { clinical study }\end{array}$} & \multirow[t]{2}{*}{12 months } & \multirow{2}{*}{$\begin{array}{l}\text { Paroxysmal, symptomatic atrial fibrillation } \geq 6 \\
\text { months refractory to } \geq 1 \text { class } 1-3 \text { antiarrhythmic } \\
\text { drugs and treated with catheter ablation }\end{array}$} & $\begin{array}{l}600 \mathrm{mg} / \text { day } \\
\alpha \text {-lipoic acid }\end{array}$ & 33 & $58.8 \pm 6.7$ & $15(45)$ \\
\hline & & & & & Placebo & 40 & $61.5 \pm 8.1$ & $23(58)$ \\
\hline \multirow{2}{*}{$\begin{array}{l}\text { Scaramuzza, } \\
2015 \text { [77] }\end{array}$} & \multirow[t]{2}{*}{ Italy } & \multirow{2}{*}{$\begin{array}{l}\text { Randomized, double-blind, } \\
\text { placebo-controlled, parallel-group, } \\
\text { pilot clinical study }\end{array}$} & \multirow{2}{*}{6 months } & \multirow{2}{*}{$\begin{array}{c}\text { Type } 1 \text { diabetes } \\
\text { endothelial dysfunction }\end{array}$} & $\begin{array}{l}800 \mathrm{mg} / \text { day } \\
\alpha \text {-lipoic acid }\end{array}$ & 25 & $16.1 \pm 3.1$ & $15(60)$ \\
\hline & & & & & Placebo & 27 & $16 \pm 3.4$ & $16(59)$ \\
\hline \multirow[t]{2}{*}{ Sola, 2005 [78] } & \multirow{2}{*}{$\begin{array}{l}\text { United Stated of } \\
\text { America }\end{array}$} & \multirow{2}{*}{$\begin{array}{l}\text { Randomized, double-blind, } \\
\text { placebo-controlled, parallel-group, } \\
\text { clinical study }\end{array}$} & \multirow[t]{2}{*}{4 weeks } & \multirow[t]{2}{*}{ Metabolic syndrome } & $\begin{array}{l}300 \mathrm{mg} / \text { day } \\
\alpha \text {-lipoic acid }\end{array}$ & 15 & $46 \pm 15$ & $5(33)$ \\
\hline & & & & & Placebo & 14 & $44 \pm 13$ & $6(43)$ \\
\hline \multirow[t]{2}{*}{ Spain, 2017 [79] } & \multirow{2}{*}{$\begin{array}{l}\text { United Stated of } \\
\text { America }\end{array}$} & \multirow{2}{*}{$\begin{array}{l}\text { Randomized, double-blind, } \\
\text { placebo-controlled, parallel-group, } \\
\text { clinical study }\end{array}$} & 2 years & Multiple sclerosis disability progression in & $\begin{array}{l}1200 \mathrm{mg} / \text { day } \\
\alpha \text {-lipoic acid }\end{array}$ & 27 & $57.9 \pm 6.7$ & $11(41)$ \\
\hline & & & & & Placebo & 24 & $59.7 \pm 6$ & $9(38)$ \\
\hline Sun, 2012 [80] & China & $\begin{array}{c}\text { Randomized, blind, } \\
\text { placebo-controlled, parallel-group, }\end{array}$ & 3 months & Dry form of age-related macular degeneration & $\begin{array}{l}600 \mathrm{mg} / \text { day } \\
\alpha \text {-lipoic acid }\end{array}$ & 32 & $65.8 \pm 7.9$ & $11(35)$ \\
\hline & & clinical study & & & Placebo & 30 & $64.5 \pm 8.1$ & $10(33)$ \\
\hline Tromba, & Italy & $\begin{array}{l}\text { Randomized, double-blind, } \\
\text { placebo-controlled parallel-group, }\end{array}$ & 12 weeks & BMI $\geq 85$ th percentile for age and sex & $\begin{array}{l}800 \mathrm{mg} / \mathrm{day} \\
\alpha \text {-lipoic acid }\end{array}$ & 34 & $11.5 \pm 1.9 *$ & $16(50) *$ \\
\hline & & clinical study & & & Placebo & 33 & $11.1 \pm 2.1^{*}$ & $20(63) *$ \\
\hline Udupa, & India & $\begin{array}{l}\text { Randomized, double-blind, } \\
\text { placebo-controlled, parallel-group, }\end{array}$ & 90 days & Type 2 diabetes mellitus & $\begin{array}{l}300 \mathrm{mg} / \text { day } \\
\alpha \text {-lipoic acid }\end{array}$ & 25 & $53.5 \pm 1.4$ & $12(48)$ \\
\hline & & clinical study & & $\mathrm{FGP} \geq 110 \mathrm{mg} / \mathrm{dL}$ and $\leq 250 \mathrm{mg} / \mathrm{dL}$ & Placebo & 25 & $53.8 \pm 2.1$ & $15(60)$ \\
\hline Vincent, & United States of & $\begin{array}{l}\text { Randomized, double-blind, } \\
\text { placebo-controlled, parallel-group, }\end{array}$ & 3 months & $\mathrm{ABI} \geq 0.3$ and $\leq 0.9$ & $\begin{array}{l}600 \mathrm{mg} / \text { day } \\
\alpha \text {-lipoic acid }\end{array}$ & 16 & $75.1 \pm 8.2$ & $9(56)$ \\
\hline & America & 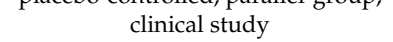 & & claudication pain with walking & Placebo & 12 & $70.7 \pm 18.9$ & $6(50)$ \\
\hline
\end{tabular}


Table 1. Cont.

\begin{tabular}{|c|c|c|c|c|c|c|c|c|}
\hline Author, Year & Location & Study Design & $\begin{array}{l}\text { Treatment } \\
\text { Duration }\end{array}$ & $\begin{array}{l}\text { Main Inclusion Criteria and } \\
\text { Underlying Disease }\end{array}$ & Study Group & $\begin{array}{l}\text { Enrolled } \\
\text { Subjects } \\
\text { (n) }\end{array}$ & $\begin{array}{c}\text { Age } \\
\text { (years; } \\
\text { mean } \pm \text { SD) }\end{array}$ & $\begin{array}{c}\text { Male } \\
{[n(\%)]}\end{array}$ \\
\hline \multirow{3}{*}{ Yadav, 2005 [84] } & \multirow{3}{*}{$\begin{array}{l}\text { United States of } \\
\text { America }\end{array}$} & \multirow{3}{*}{$\begin{array}{l}\text { Randomized, double-blind, } \\
\text { placebo-controlled, parallel-group, } \\
\text { pilot clinical study }\end{array}$} & \multirow{3}{*}{14 days } & \multirow{3}{*}{$\begin{array}{c}\text { Multiple sclerosis } \\
\text { EDSS score } \leq 7.5\end{array}$} & $\begin{array}{l}2400 \mathrm{mg} / \mathrm{day} \\
\alpha \text {-lipoic acid }\end{array}$ & 8 & $44.5(34-56)^{\S}$ & $0(0)$ \\
\hline & & & & & $\begin{array}{l}1200 \mathrm{mg} / \text { day } \\
\alpha \text {-lipoic acid }\end{array}$ & 16 & NA & $2(13)$ \\
\hline & & & & & Placebo & 9 & $50(36-66)^{\S}$ & $2(22)$ \\
\hline \multirow{2}{*}{ Yan, 2013 [85] } & \multirow{2}{*}{ China } & \multirow{2}{*}{$\begin{array}{l}\text { Randomized, double-blind, } \\
\text { placebo-controlled, crossover, } \\
\text { clinical study }\end{array}$} & \multirow{2}{*}{8 weeks } & \multirow{2}{*}{$\begin{array}{l}\mathrm{BMI} \geq 25 \mathrm{~kg} / \mathrm{m}^{2} \\
\geq 1 \text { of borderline hypertension, dyslipidemia, or } \\
\text { impaired FPG }\end{array}$} & $\begin{array}{l}1200 \mathrm{mg} / \text { day } \\
\alpha \text {-lipoic acid }\end{array}$ & \multirow{2}{*}{103} & \multirow{2}{*}{ NA } & \multirow{2}{*}{ NA } \\
\hline & & & & & Placebo & & & \\
\hline \multirow{2}{*}{$\begin{array}{l}\text { Zembron-Lacny, } \\
2013 \text { [86] }\end{array}$} & \multirow{2}{*}{ Poland } & \multirow{2}{*}{$\begin{array}{l}\text { Randomized, double-blind, } \\
\text { placebo-controlled, crossover, } \\
\text { clinical study }\end{array}$} & \multirow[t]{2}{*}{10 days } & \multirow[t]{2}{*}{ Healthy status } & $\begin{array}{l}1200 \mathrm{mg} / \text { day } \\
\alpha \text {-lipoic acid }\end{array}$ & \multirow[t]{2}{*}{16} & \multirow[t]{2}{*}{$20.7 \pm 0.9$} & \multirow[t]{2}{*}{$16(100)$} \\
\hline & & & & & Placebo & & & \\
\hline \multirow[t]{2}{*}{$\begin{array}{l}\text { Zembron-Lacny, } \\
2009[87]\end{array}$} & \multirow[t]{2}{*}{ Poland } & \multirow{2}{*}{$\begin{array}{l}\text { Randomized, double-blind, } \\
\text { placebo-controlled, crossover, } \\
\text { clinical study }\end{array}$} & \multirow[t]{2}{*}{8 days } & \multirow{2}{*}{$\begin{array}{l}\text { Physical education students } \\
\text { healthy status } \\
\text { forced training experience } \\
\geq 3 \text { years }\end{array}$} & $\begin{array}{l}1200 \mathrm{mg} / \text { day } \\
\alpha \text {-lipoic acid }\end{array}$ & \multirow[t]{2}{*}{13} & \multirow[t]{2}{*}{$25.5 \pm 6$} & \multirow[t]{2}{*}{$13(100$} \\
\hline & & & & & Placebo & & & \\
\hline
\end{tabular}

Type 1 or 2 diabetes (duration $\geq 1$ year) tage 1 or 2 a distal symmetric sensorimotor polyneuropathy due to diabetes

$$
\text { stable insulin regimen }
$$

Canada, Croatia, Randomized, double-blind,

Ziegler, Denmark, placebo-controlled, parallel-group,

2011 [88] France, Italy,

Spain,

The Netherlands,

United

Kingdom,
United States of

clinical study

4 years$$
\mathrm{NIS}_{[\mathrm{LL}]}+7 \geq 2
$$

one of the following abnormalities: abnorma

nerve conduction attributes in two separate

nerves $\geq 99$ th percentile for distal latency or

$\leq 1$ st percentile for nerve conduction velocity or

amplitude OR HRBD $\geq 1$ st percentile or TSS in the feet $<5$
$600 \mathrm{mg} /$ day

$\alpha$-lipoic acid

23

$53.3 \pm 8.3$

$152(66)$

America 
Table 1. Cont.

\begin{tabular}{|c|c|c|c|c|c|c|c|c|}
\hline Author, Year & Location & Study Design & $\begin{array}{l}\text { Treatment } \\
\text { Duration }\end{array}$ & $\begin{array}{l}\text { Main Inclusion Criteria and } \\
\text { Underlying Disease }\end{array}$ & Study Group & $\begin{array}{l}\text { Enrolled } \\
\text { Subjects } \\
\text { (n) }\end{array}$ & $\begin{array}{c}\text { Age } \\
(\text { years; } \\
\text { mean } \pm \text { SD) }\end{array}$ & $\begin{array}{c}\text { Male } \\
{[n(\%)]}\end{array}$ \\
\hline \multirow{4}{*}{$\begin{array}{l}\text { Ziegler, } \\
2006[89]\end{array}$} & \multirow{4}{*}{$\begin{array}{l}\text { Israel and } \\
\text { Russia }\end{array}$} & \multirow{4}{*}{$\begin{array}{l}\text { Randomized, double-blind, } \\
\text { placebo-controlled, parallel-group, } \\
\text { clinical study }\end{array}$} & \multirow{4}{*}{5 weeks } & \multirow{4}{*}{$\begin{array}{c}\text { Type } 1 \text { or } 2 \text { diabetes } \\
\text { HbA1c }<10 \% \\
\text { symptomatic distal symmetric polyneuropathy } \\
\text { due to diabetes } \\
\text { TSS }>7.5 \\
\text { NIS }[\text { [LL] } \geq 2 \\
\text { pin-prick test }\end{array}$} & $\begin{array}{l}1800 \mathrm{mg} / \text { day } \\
\alpha \text {-lipoic acid }\end{array}$ & 46 & $59 \pm 9$ & $19(41)$ \\
\hline & & & & & $\begin{array}{l}1200 \mathrm{mg} / \text { day } \\
\alpha \text {-lipoic acid }\end{array}$ & 47 & $59 \pm 12$ & $19(40)$ \\
\hline & & & & & $\begin{array}{l}600 \mathrm{mg} / \mathrm{day} \\
\alpha \text {-lipoic acid }\end{array}$ & 45 & $56 \pm 12$ & $20(44)$ \\
\hline & & & & & Placebo & 43 & $57 \pm 11$ & $15(35)$ \\
\hline
\end{tabular}




\subsection{Risk of Bias Assessment}

Almost all of the included studies were characterized by sufficient information regarding sequence generation, allocation concealment, personal and outcome assessments, incomplete outcome data, and selective outcome reporting. Details of the quality of bias assessment are reported in Table 2.

Table 2. Quality of bias assessment of the included studies according to Cochrane guidelines.

\begin{tabular}{|c|c|c|c|c|c|c|}
\hline Author, Year & $\begin{array}{l}\text { Sequence } \\
\text { Generation }\end{array}$ & $\begin{array}{l}\text { Allocation } \\
\text { Concealment }\end{array}$ & $\begin{array}{l}\text { Blinding to } \\
\text { Participants, } \\
\text { Personnel and } \\
\text { Outcome } \\
\text { Assessment }\end{array}$ & $\begin{array}{c}\text { Incomplete } \\
\text { Outcome } \\
\text { Data }\end{array}$ & $\begin{array}{l}\text { Selective } \\
\text { Outcome } \\
\text { Reporting }\end{array}$ & $\begin{array}{c}\text { Other } \\
\text { Potential } \\
\text { Threats to } \\
\text { Validity }\end{array}$ \\
\hline $\begin{array}{l}\text { Ahmadi, } \\
2013 \text { [20] }\end{array}$ & $\mathrm{L}$ & $\mathrm{L}$ & $\mathrm{H}$ & $\mathrm{L}$ & $\mathrm{L}$ & $\mathrm{U}$ \\
\hline Ansar, 2011 [21] & $\mathrm{L}$ & $\mathrm{L}$ & $\mathrm{L}$ & $\mathrm{L}$ & $\mathrm{U}$ & $\mathrm{L}$ \\
\hline $\begin{array}{c}\text { Aslfalah, } \\
\text { 2019a [22] }\end{array}$ & $\mathrm{L}$ & $\mathrm{L}$ & $\mathrm{L}$ & $\mathrm{L}$ & $\mathrm{L}$ & $\mathrm{L}$ \\
\hline $\begin{array}{c}\text { Aslfalah, } \\
\text { 2019b [23] }\end{array}$ & $\mathrm{L}$ & $\mathrm{L}$ & $\mathrm{L}$ & $\mathrm{L}$ & $\mathrm{L}$ & $\mathrm{L}$ \\
\hline $\begin{array}{c}\text { Baumgartner, } \\
2017 \text { [24] }\end{array}$ & $\mathrm{L}$ & $\mathrm{L}$ & $\mathrm{L}$ & $\mathrm{L}$ & $\mathrm{L}$ & $\mathrm{L}$ \\
\hline Baziar, 2020 [25] & $\mathrm{L}$ & $\mathrm{L}$ & $\mathrm{L}$ & $\mathrm{L}$ & $\mathrm{L}$ & $\mathrm{L}$ \\
\hline Bobe, 2020 [26] & $\mathrm{L}$ & $\mathrm{L}$ & $\mathrm{L}$ & $\mathrm{L}$ & $\mathrm{L}$ & $\mathrm{L}$ \\
\hline $\begin{array}{c}\text { Boriani, } \\
2017 \text { [27] }\end{array}$ & $\mathrm{L}$ & $\mathrm{L}$ & $\mathrm{L}$ & $\mathrm{L}$ & $\mathrm{L}$ & $\mathrm{L}$ \\
\hline $\begin{array}{l}\text { Carbone, } \\
2009 \text { [28] }\end{array}$ & $\mathrm{L}$ & $\mathrm{L}$ & $\mathrm{L}$ & $\mathrm{L}$ & $\mathrm{L}$ & $\mathrm{L}$ \\
\hline $\begin{array}{l}\text { Cavalcanti, } \\
2009 \text { [29] }\end{array}$ & $\mathrm{L}$ & $\mathrm{L}$ & $\mathrm{L}$ & $\mathrm{L}$ & $\mathrm{L}$ & $\mathrm{L}$ \\
\hline $\begin{array}{l}\text { Durastanti, } \\
2016 \text { [30] }\end{array}$ & $\mathrm{L}$ & $\mathrm{L}$ & $\mathrm{L}$ & $\mathrm{U}$ & $\mathrm{U}$ & $\mathrm{U}$ \\
\hline $\begin{array}{l}\text { El Amrousy, } \\
2020 \text { [31] }\end{array}$ & $\mathrm{L}$ & $\mathrm{L}$ & $\mathrm{L}$ & $\mathrm{L}$ & $\mathrm{L}$ & $\mathrm{L}$ \\
\hline $\begin{array}{l}\text { Falardeau, } \\
2019 \text { [32] }\end{array}$ & $\mathrm{L}$ & $\mathrm{L}$ & $\mathrm{L}$ & L & $\mathrm{L}$ & $\mathrm{L}$ \\
\hline $\begin{array}{l}\text { Femiano, } \\
2002[33]\end{array}$ & $\mathrm{U}$ & $\mathrm{L}$ & $\mathrm{L}$ & $\mathrm{L}$ & $\mathrm{U}$ & $\mathrm{U}$ \\
\hline $\begin{array}{c}\text { Georgakouli, } \\
2018 \text { [34] }\end{array}$ & $\mathrm{L}$ & $\mathrm{L}$ & $\mathrm{L}$ & $\mathrm{L}$ & $\mathrm{L}$ & $\mathrm{L}$ \\
\hline $\begin{array}{l}\text { Gianturco, } \\
2009 \text { [35] }\end{array}$ & $\mathrm{L}$ & $\mathrm{L}$ & $\mathrm{L}$ & $\mathrm{L}$ & $\mathrm{U}$ & $\mathrm{L}$ \\
\hline Gilron, 2020 [36] & $\mathrm{L}$ & $\mathrm{L}$ & $\mathrm{L}$ & $\mathrm{L}$ & $\mathrm{L}$ & $\mathrm{L}$ \\
\hline $\begin{array}{l}\text { Gosselin, } \\
2019 \text { [37] }\end{array}$ & $\mathrm{L}$ & $\mathrm{L}$ & $\mathrm{L}$ & $\mathrm{L}$ & $\mathrm{L}$ & $\mathrm{L}$ \\
\hline Guo, 2014 [38] & $\mathrm{L}$ & $\mathrm{L}$ & $\mathrm{L}$ & $\mathrm{L}$ & $\mathrm{L}$ & $\mathrm{L}$ \\
\hline $\begin{array}{c}\text { Haghighian, } \\
2015 \text { [39] }\end{array}$ & $\mathrm{L}$ & $\mathrm{L}$ & $\mathrm{L}$ & $\mathrm{L}$ & $\mathrm{L}$ & $\mathrm{L}$ \\
\hline Hejazi, 2018 [40] & $\mathrm{L}$ & $\mathrm{L}$ & $\mathrm{L}$ & $\mathrm{L}$ & $\mathrm{L}$ & $\mathrm{L}$ \\
\hline Huang, 2008 [41] & $\mathrm{L}$ & $\mathrm{L}$ & $\mathrm{L}$ & $\mathrm{L}$ & $\mathrm{L}$ & $\mathrm{L}$ \\
\hline $\begin{array}{c}\text { Huerta, } \\
2016 \text { [42] }\end{array}$ & $\mathrm{L}$ & $\mathrm{L}$ & $\mathrm{L}$ & $\mathrm{L}$ & $\mathrm{L}$ & $\mathrm{L}$ \\
\hline $\begin{array}{l}\text { Huerta, } \\
2015 \text { [43] }\end{array}$ & $\mathrm{L}$ & $\mathrm{L}$ & $\mathrm{L}$ & $\mathrm{L}$ & $\mathrm{L}$ & $\mathrm{L}$ \\
\hline
\end{tabular}


Table 2. Cont.

\begin{tabular}{|c|c|c|c|c|c|c|}
\hline Author, Year & $\begin{array}{l}\text { Sequence } \\
\text { Generation }\end{array}$ & $\begin{array}{l}\text { Allocation } \\
\text { Concealment }\end{array}$ & $\begin{array}{l}\text { Blinding to } \\
\text { Participants, } \\
\text { Personnel and } \\
\text { Outcome } \\
\text { Assessment }\end{array}$ & $\begin{array}{l}\text { Incomplete } \\
\text { Outcome } \\
\text { Data }\end{array}$ & $\begin{array}{l}\text { Selective } \\
\text { Outcome } \\
\text { Reporting }\end{array}$ & $\begin{array}{c}\text { Other } \\
\text { Potential } \\
\text { Threats to } \\
\text { Validity }\end{array}$ \\
\hline Jacob, 1999 [44] & $\mathrm{L}$ & $\mathrm{L}$ & $\mathrm{L}$ & $\mathrm{L}$ & $\mathrm{U}$ & $\mathrm{H}$ \\
\hline $\begin{array}{l}\text { Jamshidi, } \\
2020 \text { [45] }\end{array}$ & $\mathrm{L}$ & $\mathrm{L}$ & $\mathrm{L}$ & $\mathrm{L}$ & $\mathrm{L}$ & $\mathrm{L}$ \\
\hline $\begin{array}{l}\text { Jariwalla, } \\
2008 \text { [46] }\end{array}$ & $\mathrm{L}$ & $\mathrm{L}$ & $\mathrm{L}$ & $\mathrm{L}$ & $\mathrm{U}$ & $\mathrm{H}$ \\
\hline $\begin{array}{l}\text { Khabbazi, } \\
2012 \text { [47] }\end{array}$ & $\mathrm{L}$ & $\mathrm{L}$ & $\mathrm{L}$ & $\mathrm{L}$ & $\mathrm{L}$ & $\mathrm{L}$ \\
\hline Khalili, 2017 [48] & $\mathrm{L}$ & $\mathrm{L}$ & $\mathrm{L}$ & $\mathrm{L}$ & $\mathrm{L}$ & $\mathrm{L}$ \\
\hline Khalili, 2014 [49] & $\mathrm{L}$ & $\mathrm{L}$ & $\mathrm{L}$ & $\mathrm{L}$ & $\mathrm{L}$ & $\mathrm{L}$ \\
\hline Kim, 2020 [50] & $\mathrm{L}$ & $\mathrm{L}$ & $\mathrm{L}$ & $\mathrm{L}$ & $\mathrm{L}$ & $\mathrm{L}$ \\
\hline Kim, 2016 [51] & $\mathrm{L}$ & $\mathrm{L}$ & $\mathrm{L}$ & $\mathrm{L}$ & $\mathrm{L}$ & $\mathrm{L}$ \\
\hline Koh, 2011 [52] & $\mathrm{L}$ & $\mathrm{L}$ & $\mathrm{L}$ & L & $\mathrm{L}$ & $\mathrm{L}$ \\
\hline $\begin{array}{l}\text { Lampitella, } \\
2005 \text { [53] }\end{array}$ & $\mathrm{L}$ & $\mathrm{U}$ & $\mathrm{U}$ & $\mathrm{L}$ & $\mathrm{L}$ & $\mathrm{U}$ \\
\hline Lee, 2017 [54] & $\mathrm{L}$ & $\mathrm{L}$ & $\mathrm{L}$ & $\mathrm{L}$ & $\mathrm{L}$ & $\mathrm{L}$ \\
\hline Loy, 2018 [55] & $\mathrm{L}$ & $\mathrm{L}$ & $\mathrm{L}$ & $\mathrm{L}$ & $\mathrm{L}$ & $\mathrm{L}$ \\
\hline $\begin{array}{c}\text { López- } \\
\text { D'Alessandro, } \\
2011 \text { [56] }\end{array}$ & $\mathrm{L}$ & $\mathrm{L}$ & $\mathrm{L}$ & $\mathrm{H}$ & $\mathrm{H}$ & $\mathrm{U}$ \\
\hline $\begin{array}{c}\text { López-Jornet, } \\
2009 \text { [57] }\end{array}$ & $\mathrm{L}$ & $\mathrm{L}$ & $\mathrm{L}$ & $\mathrm{L}$ & $\mathrm{L}$ & $\mathrm{L}$ \\
\hline Magis, 2007 [58] & $\mathrm{L}$ & $\mathrm{L}$ & $\mathrm{L}$ & $\mathrm{L}$ & $\mathrm{L}$ & $\mathrm{L}$ \\
\hline $\begin{array}{l}\text { Manning, } \\
2013 \text { [59] }\end{array}$ & $\mathrm{L}$ & $\mathrm{L}$ & $\mathrm{L}$ & $\mathrm{L}$ & $\mathrm{L}$ & $\mathrm{L}$ \\
\hline $\begin{array}{l}\text { Marfella, } \\
2016 \text { [60] }\end{array}$ & $\mathrm{L}$ & $\mathrm{L}$ & $\mathrm{U}$ & $\mathrm{L}$ & $\mathrm{L}$ & $\mathrm{U}$ \\
\hline $\begin{array}{l}\text { Marshall, } \\
1982 \text { [61] }\end{array}$ & $\mathrm{L}$ & $\mathrm{L}$ & $\mathrm{L}$ & $\mathrm{L}$ & $\mathrm{L}$ & $\mathrm{L}$ \\
\hline $\begin{array}{l}\text { Martins, } \\
2009 \text { [62] }\end{array}$ & $\mathrm{L}$ & $\mathrm{L}$ & $\mathrm{U}$ & $\mathrm{L}$ & $\mathrm{L}$ & $\mathrm{U}$ \\
\hline $\begin{array}{l}\text { Mendes, } \\
2014 \text { [63] }\end{array}$ & $\mathrm{L}$ & $\mathrm{L}$ & $\mathrm{L}$ & $\mathrm{L}$ & $\mathrm{H}$ & $\mathrm{U}$ \\
\hline $\begin{array}{c}\text { Mendoza- } \\
\text { Núñez, } 2019[64]\end{array}$ & $\mathrm{L}$ & $\mathrm{L}$ & $\mathrm{L}$ & $\mathrm{L}$ & $\mathrm{L}$ & $\mathrm{L}$ \\
\hline $\begin{array}{l}\text { Mirtaheri, } \\
2014 \text { [65] }\end{array}$ & $\mathrm{L}$ & $\mathrm{L}$ & $\mathrm{L}$ & $\mathrm{L}$ & $\mathrm{L}$ & $\mathrm{L}$ \\
\hline $\begin{array}{l}\text { Mohammadi, } \\
2018 \text { [66] }\end{array}$ & $\mathrm{L}$ & $\mathrm{L}$ & $\mathrm{L}$ & $\mathrm{L}$ & $\mathrm{L}$ & $\mathrm{L}$ \\
\hline $\begin{array}{l}\text { Mohammadi, } \\
2015 \text { [67] }\end{array}$ & $\mathrm{L}$ & $\mathrm{L}$ & $\mathrm{L}$ & $\mathrm{L}$ & $\mathrm{L}$ & $\mathrm{L}$ \\
\hline Mollo, 2012 [68] & $\mathrm{L}$ & $\mathrm{L}$ & $\mathrm{L}$ & $\mathrm{L}$ & $\mathrm{L}$ & $\mathrm{L}$ \\
\hline $\begin{array}{l}\text { Monroy Guízar, } \\
2018 \text { [69] }\end{array}$ & $\mathrm{L}$ & $\mathrm{L}$ & $\mathrm{L}$ & $\mathrm{L}$ & $\mathrm{L}$ & $\mathrm{L}$ \\
\hline $\begin{array}{l}\text { Palacios- } \\
\text { Sánchez, } \\
2015 \text { [70] }\end{array}$ & $\mathrm{L}$ & $\mathrm{L}$ & $\mathrm{L}$ & $\mathrm{L}$ & $\mathrm{L}$ & $\mathrm{L}$ \\
\hline
\end{tabular}


Table 2. Cont.

\begin{tabular}{|c|c|c|c|c|c|c|}
\hline Author, Year & $\begin{array}{l}\text { Sequence } \\
\text { Generation }\end{array}$ & $\begin{array}{l}\text { Allocation } \\
\text { Concealment }\end{array}$ & $\begin{array}{l}\text { Blinding to } \\
\text { Participants, } \\
\text { Personnel and } \\
\text { Outcome } \\
\text { Assessment }\end{array}$ & $\begin{array}{l}\text { Incomplete } \\
\text { Outcome } \\
\text { Data }\end{array}$ & $\begin{array}{l}\text { Selective } \\
\text { Outcome } \\
\text { Reporting }\end{array}$ & $\begin{array}{c}\text { Other } \\
\text { Potential } \\
\text { Threats to } \\
\text { Validity }\end{array}$ \\
\hline $\begin{array}{c}\text { Porasuphatana, } \\
\quad 2012[71]\end{array}$ & $\mathrm{L}$ & $\mathrm{L}$ & $\mathrm{L}$ & $\mathrm{L}$ & $\mathrm{L}$ & $\mathrm{H}$ \\
\hline $\begin{array}{c}\text { Pourghasem } \\
\text { Gargari, } \\
2014 \text { [72] }\end{array}$ & $\mathrm{L}$ & $\mathrm{L}$ & $\mathrm{L}$ & $\mathrm{L}$ & $\mathrm{L}$ & $\mathrm{L}$ \\
\hline $\begin{array}{l}\text { Rahmanabadi, } \\
2019 \text { [4] }\end{array}$ & $\mathrm{L}$ & $\mathrm{L}$ & $\mathrm{L}$ & $\mathrm{L}$ & $\mathrm{L}$ & $\mathrm{L}$ \\
\hline $\begin{array}{l}\text { Ruhnau, } \\
1999 \text { [73] }\end{array}$ & $\mathrm{L}$ & $\mathrm{L}$ & $\mathrm{L}$ & $\mathrm{L}$ & $\mathrm{L}$ & $\mathrm{L}$ \\
\hline Safa, 2014 [74] & $\mathrm{L}$ & $\mathrm{L}$ & $\mathrm{L}$ & $\mathrm{L}$ & $\mathrm{L}$ & $\mathrm{L}$ \\
\hline $\begin{array}{l}\text { Sammour, } \\
2019 \text { [75] }\end{array}$ & $\mathrm{L}$ & $\mathrm{L}$ & $\mathrm{L}$ & $\mathrm{L}$ & $\mathrm{L}$ & $\mathrm{L}$ \\
\hline Sardu, 2017 [76] & $\mathrm{L}$ & $\mathrm{L}$ & $\mathrm{L}$ & $\mathrm{L}$ & $\mathrm{L}$ & $\mathrm{L}$ \\
\hline $\begin{array}{c}\text { Scaramuzza, } \\
2015 \text { [77] }\end{array}$ & $\mathrm{L}$ & $\mathrm{L}$ & $\mathrm{L}$ & $\mathrm{L}$ & $\mathrm{L}$ & $\mathrm{L}$ \\
\hline Sola, 2005 [78] & $\mathrm{L}$ & $\mathrm{L}$ & $\mathrm{L}$ & $\mathrm{L}$ & $\mathrm{L}$ & $\mathrm{L}$ \\
\hline Spain, 2017 [79] & $\mathrm{L}$ & $\mathrm{L}$ & $\mathrm{L}$ & $\mathrm{L}$ & $\mathrm{L}$ & $\mathrm{L}$ \\
\hline Sun, 2012 [80] & $\mathrm{L}$ & $\mathrm{U}$ & $\mathrm{U}$ & $\mathrm{L}$ & $\mathrm{L}$ & $\mathrm{U}$ \\
\hline $\begin{array}{r}\text { Tromba, } \\
2019 \text { [81] }\end{array}$ & $\mathrm{L}$ & $\mathrm{L}$ & $\mathrm{L}$ & $\mathrm{L}$ & $\mathrm{L}$ & $\mathrm{L}$ \\
\hline $\begin{array}{l}\text { Udupa, } \\
2013 \text { [82] }\end{array}$ & $\mathrm{L}$ & $\mathrm{L}$ & $\mathrm{L}$ & $\mathrm{L}$ & $\mathrm{L}$ & $\mathrm{L}$ \\
\hline $\begin{array}{l}\text { Vincent, } \\
2007 \text { [83] }\end{array}$ & $\mathrm{L}$ & $\mathrm{L}$ & $\mathrm{L}$ & $\mathrm{L}$ & $\mathrm{L}$ & $\mathrm{L}$ \\
\hline Yadav, 2005 [84] & $\mathrm{L}$ & $\mathrm{L}$ & $\mathrm{L}$ & $\mathrm{L}$ & L & $\mathrm{L}$ \\
\hline Yan, 2013 [85] & $\mathrm{L}$ & $\mathrm{L}$ & $\mathrm{L}$ & $\mathrm{L}$ & $\mathrm{L}$ & $\mathrm{L}$ \\
\hline $\begin{array}{c}\text { Zembron- } \\
\text { Lacny, } 2013 \text { [86] }\end{array}$ & $\mathrm{L}$ & $\mathrm{L}$ & $\mathrm{L}$ & $\mathrm{L}$ & $\mathrm{L}$ & $\mathrm{L}$ \\
\hline $\begin{array}{c}\text { Zembron- } \\
\text { Lacny, } 2009 \text { [87] }\end{array}$ & $\mathrm{L}$ & $\mathrm{L}$ & $\mathrm{L}$ & $\mathrm{L}$ & $\mathrm{L}$ & $\mathrm{L}$ \\
\hline Ziegler, 2011 [88] & $\mathrm{L}$ & $\mathrm{L}$ & $\mathrm{L}$ & $\mathrm{L}$ & $\mathrm{L}$ & $\mathrm{L}$ \\
\hline Ziegler, 2006 [89] & $\mathrm{L}$ & $\mathrm{L}$ & $\mathrm{L}$ & $\mathrm{L}$ & $\mathrm{L}$ & $\mathrm{L}$ \\
\hline
\end{tabular}

The quality of evidence for each outcome across all the studies was considered high in accordance with the GRADE approach.

\subsection{Primary Outcomes}

\subsubsection{Hypoglycaemic Episodes}

Symptoms defined as 'similar to hypoglycaemic episodes' were reported only by Jacob et al. and were exclusively experienced by subjects randomized to placebo. Authors did not report if an attempt for treatment rechallenging was made during the trial [44]. 


\subsubsection{Gastrointestinal AEs}

Meta-analysis of extracted data suggested that supplementation with ALA was not associated with an increased risk of gastrointestinal AEs ( $\mathrm{OR}=1.32,95 \% \mathrm{CI} 0.97$ to $\left.1.78 ; p=0.073 ; \mathrm{I}^{2}=0 \%\right)$ (Figure 2). The finding was robust in the leave-one-out sensitivity analysis (Figure S1).

\section{Gastrointestinal AEs}

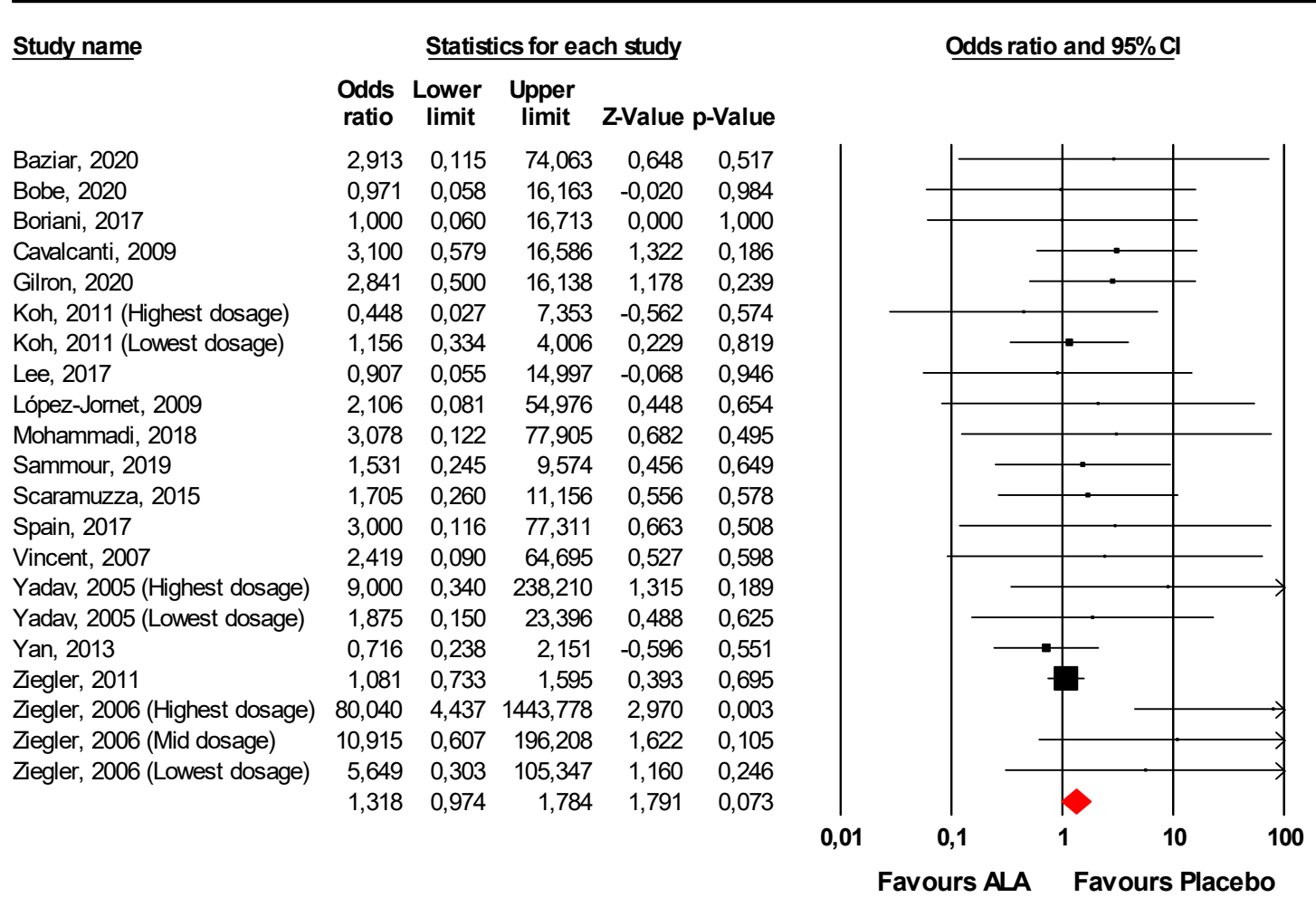

Figure 2. Forest plot for the risk of gastrointestinal adverse events (AEs) following alpha-lipoic acid (ALA) supplementation versus placebo.

Visually, the funnel plot of standard error by log OR was slightly asymmetric (Figure S2). This asymmetry was imputed to eight potentially missing studies on the left-side of the plot, which reduced the estimated effect size to 1.12 (95\% CI 0.84 to 1.49). Egger's linear regression and Begg's rank correlation confirmed the presence of publication bias for the analysis $(p<0.05)$.

\subsubsection{Neurological AEs}

Meta-analysis of extracted data suggested that supplementation with ALA was not associated with an increased risk of neurological AEs (OR $=1.53,95 \% \mathrm{CI} 0.88$ to $2.63 ; p=0.129 ; \mathrm{I}^{2}=0 \%$ ) (Figure 3). The finding was robust in the leave-one-out sensitivity analysis (Figure S3).

Visually, the funnel plot of standard error by log OR was slightly asymmetric (Figure S4). This asymmetry was imputed to 4 potentially missing studies on the left-side of the plot, which reduced the estimated effect size to 1.26 (95\% CI 0.76 to 2.10). However, neither Egger's linear regression nor Begg's rank correlation confirmed the presence of publication bias for the analysis $(p>0.05$ for both tests). 
Neurological AEs

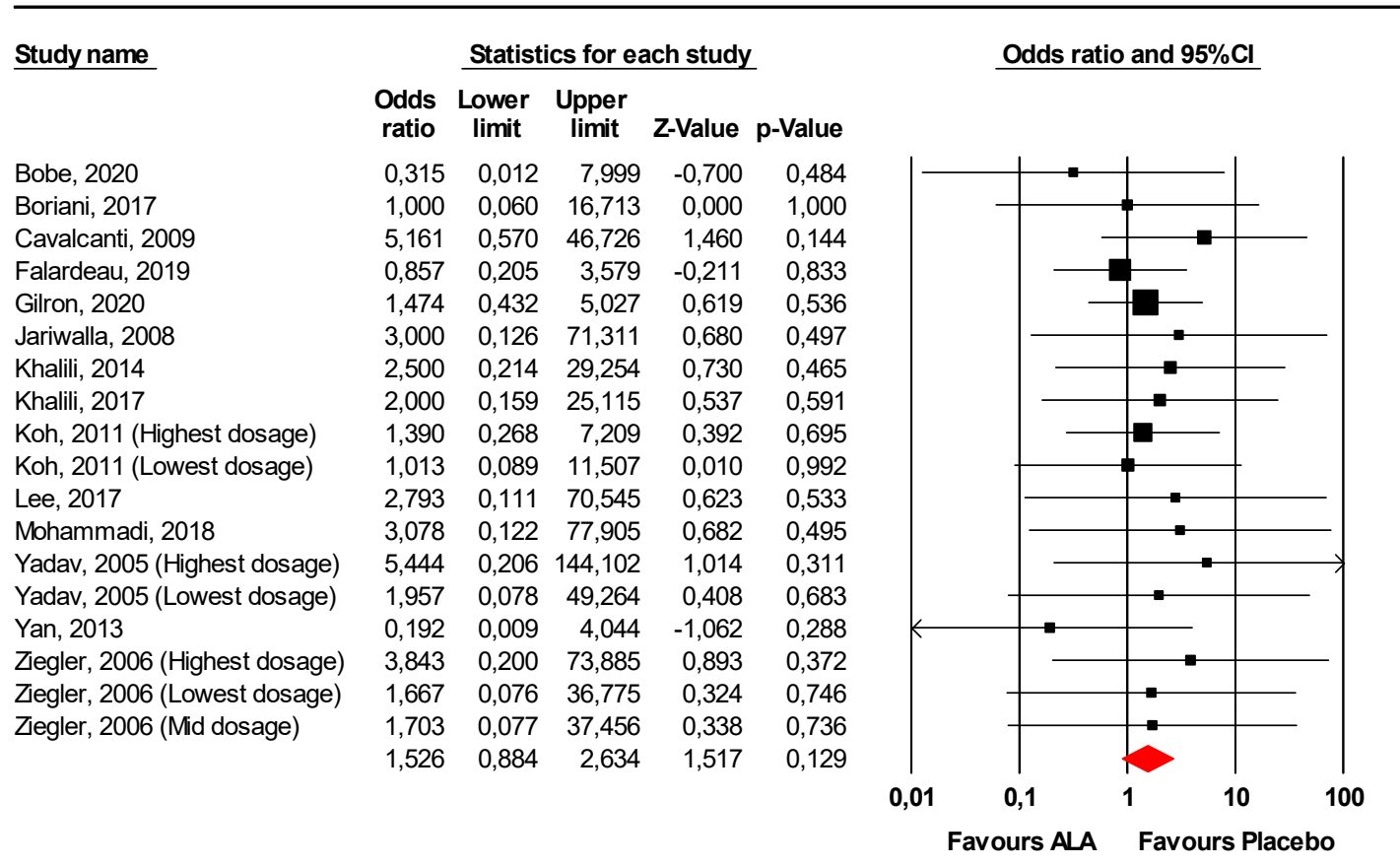

Figure 3. Forest plot for the risk of neurological AEs following ALA supplementation versus placebo.

\subsubsection{Psychiatric Disorders}

Meta-analysis of extracted data suggested that supplementation with ALA was not associated with an increased risk of psychiatric disorders ( $\mathrm{OR}=1.13,95 \% \mathrm{CI} 0.64$ to $1.99 ; p=0.668 ; \mathrm{I}^{2}=0 \%$ ) (Figure 4). The finding was robust in the leave-one-out sensitivity analysis (Figure S5).

\section{Psychiatric AEs}

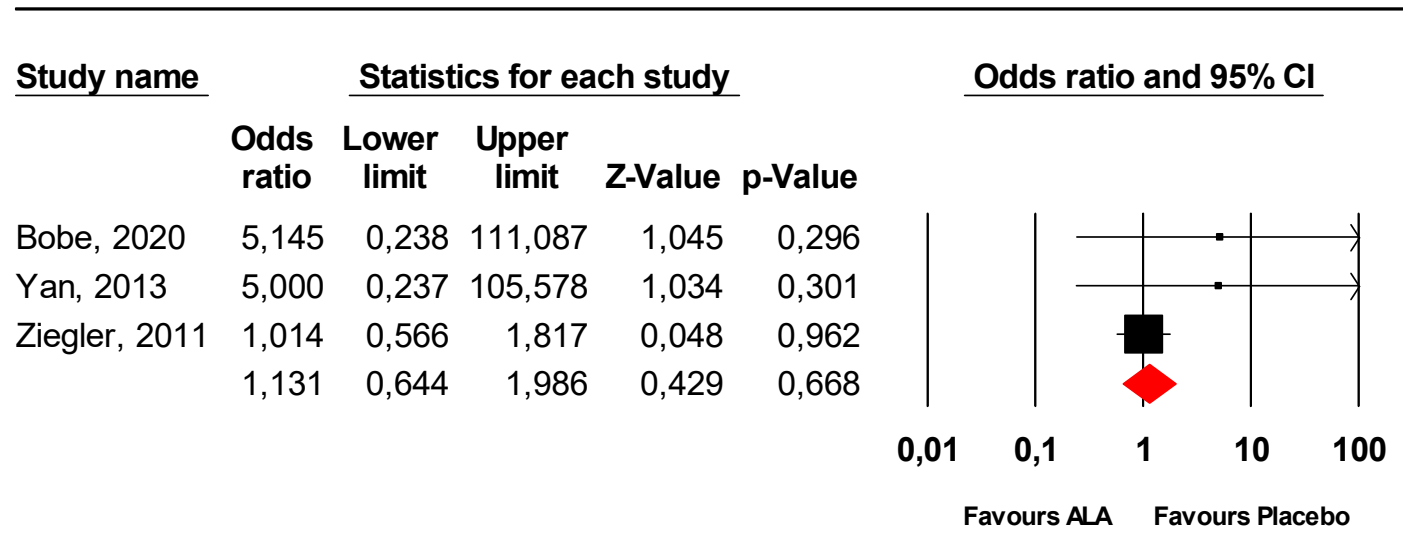

Figure 4. Forest plot for the risk of psychiatric AEs following ALA supplementation versus placebo.

Visually, the funnel plot of standard error by log OR was slightly asymmetric (Figure S6). This asymmetry was imputed to two potentially missing studies on the left-side of the plot, which reduced the estimated effect size to 1.01 (95\% CI 0.59 to 1.75). Egger's linear regression confirmed the presence of publication bias for the analysis $(p<0.01)$, though Begg's rank correlation did not.

\subsubsection{Musculoskeletal AEs}

Meta-analysis of extracted data suggested that supplementation with ALA was not associated with an increased risk of musculoskeletal AEs (OR $=0.76,95 \%$ CI 0.22 to $2.64 ; p=0.666 ; \mathrm{I}^{2}=0 \%$ ) (Figure 5). The finding was robust in the leave-one-out sensitivity analysis (Figure S7). 


\section{Musculoskeletal AEs}

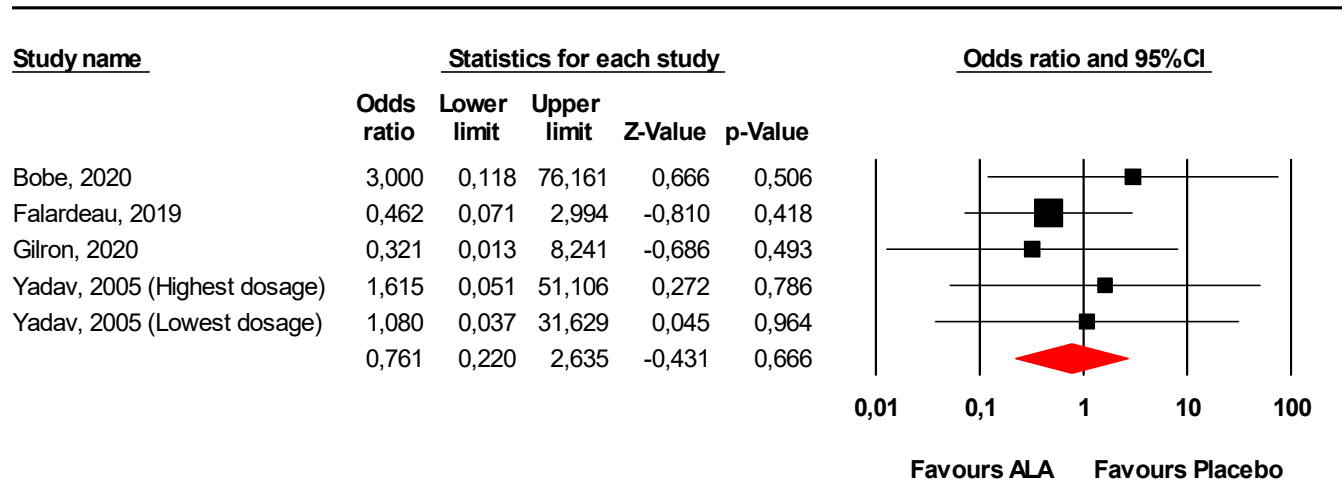

Figure 5. Forest plot for the risk of musculoskeletal AEs following ALA supplementation versus placebo.

Visually, the funnel plot of standard error by log OR was slightly asymmetric (Figure S8). This asymmetry was imputed to 2 potentially missing studies on the left-side of the plot, which reduced the estimated effect size to 0.50 ( $95 \%$ CI 0.17 to 1.51). However, neither Egger's linear regression nor Begg's rank correlation confirmed the presence of publication bias for the analysis $(p>0.05$ for both tests).

\subsubsection{Skin AEs}

Meta-analysis of extracted data suggested that supplementation with ALA was not associated with an increased risk of skin AEs (OR $=1.13,95 \%$ CI 0.82 to $1.56 ; p=0.469 ; \mathrm{I}^{2}=33.6 \%$ ) (Figure 6). The finding was robust in the leave-one-out sensitivity analysis (Figure S9).

\section{Skin AEs}

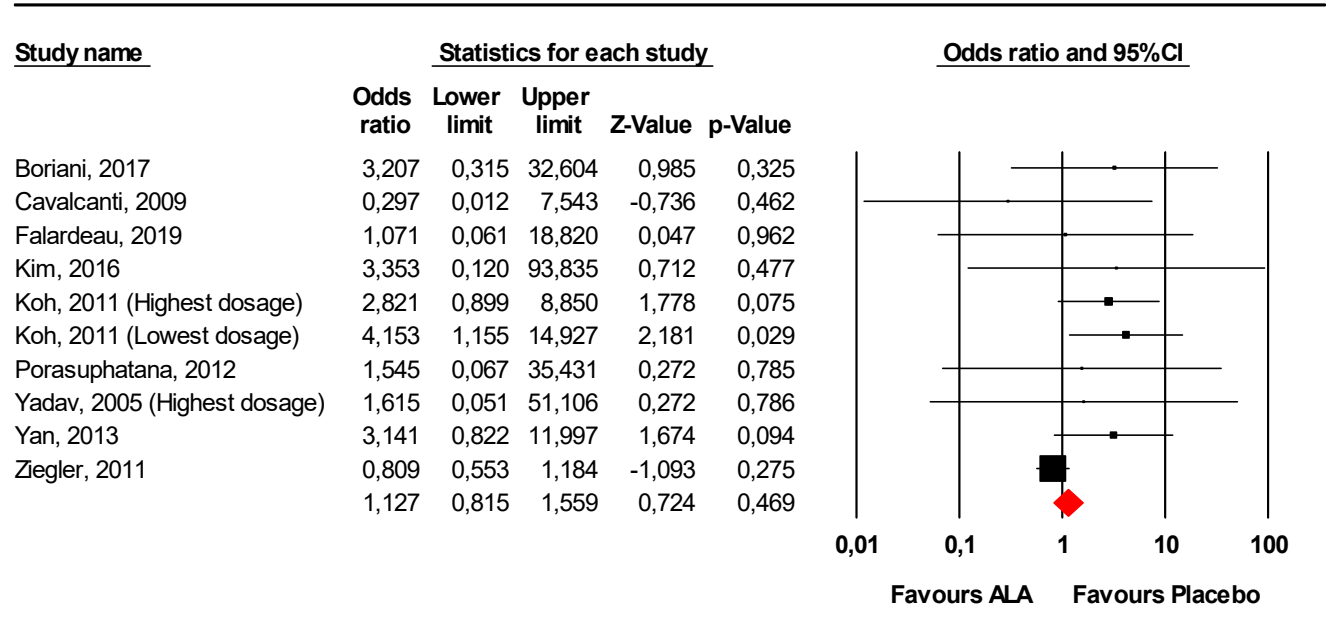

Figure 6. Forest plot for the risk of skin AEs following ALA supplementation versus placebo.

Visually, the funnel plot of standard error by $\log$ OR was slightly asymmetric (Figure S10). This asymmetry was imputed to four potentially missing studies on the left-side of the plot, which reduced the estimated effect size to 0.92 (95\% CI 0.68 to 1.24). However, neither Egger's linear regression nor Begg's rank correlation confirmed the presence of publication bias for the analysis $(p>0.05$ for both tests).

\subsubsection{Infections}

Meta-analysis of extracted data suggested that supplementation with ALA was not associated with an increased risk of infections ( $\mathrm{OR}=0.93,95 \% \mathrm{CI} 0.18$ to $4.65 ; p=0.925 ; \mathrm{I}^{2}=0 \%$ ) (Figure 7). The finding was robust in the leave-one-out sensitivity analysis (Figure S11). 


\section{Infections}

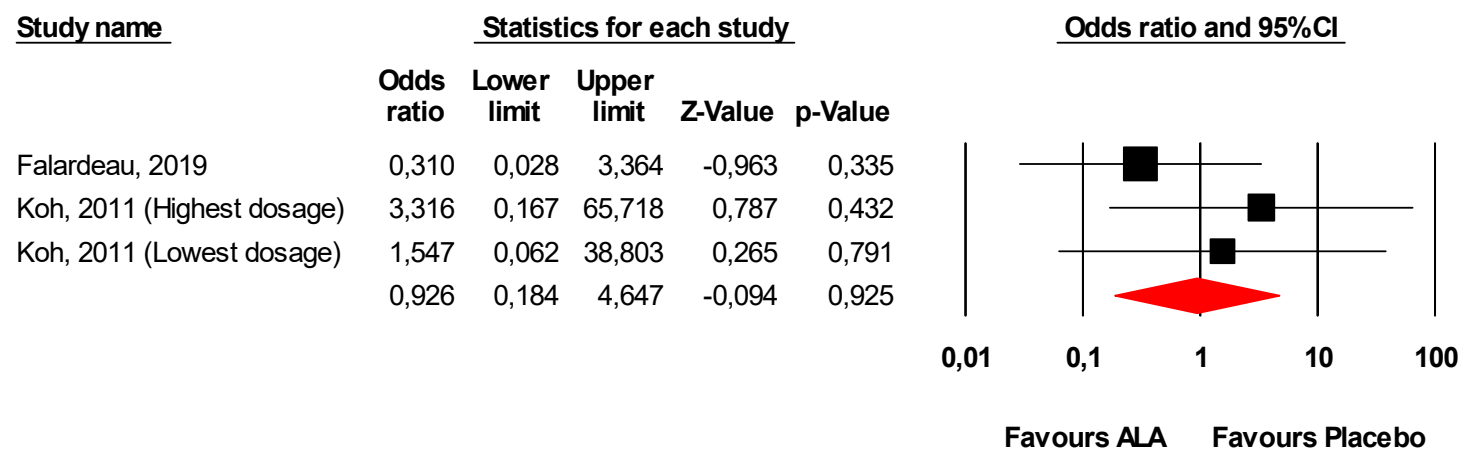

Figure 7. Forest plot for the risk of infections following ALA supplementation versus placebo.

Visually, the funnel plot of standard error by log OR was slightly asymmetric (Figure S12). This asymmetry was imputed to two potentially missing studies on the left-side of the plot, which reduced the estimated effect size to 0.31 (95\% CI 0.08 to 1.13). However, neither Egger's linear regression nor Begg's rank correlation confirmed the presence of publication bias for the analysis $(p>0.05$ for both tests).

\subsubsection{System AEs}

Meta-analysis of extracted data suggested that supplementation with ALA was not associated with an increased risk of CV system AEs (OR $=1.25,95 \%$ CI 0.84 to $1.85 ; p=0.276 ; \mathrm{I}^{2}=15.8 \%$ ) (Figure 8). The finding was robust in the leave-one-out sensitivity analysis (Figure S13).

\section{CV system AEs}

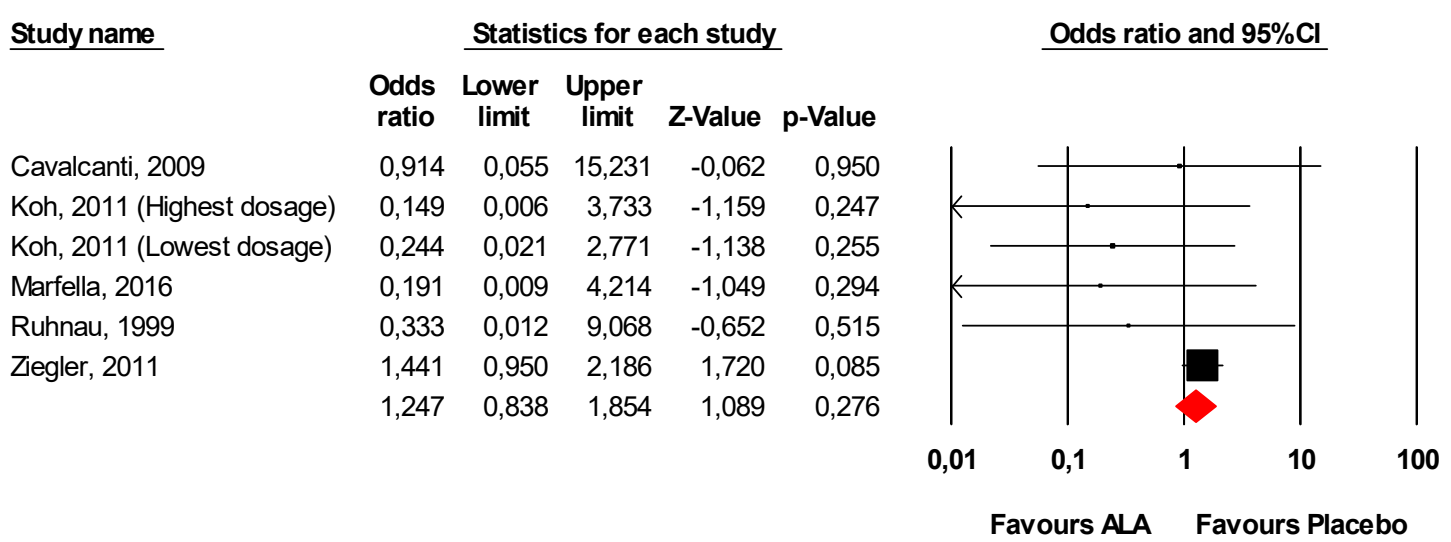

Figure 8. Forest plot for the risk of CV system AEs following ALA supplementation versus placebo.

Visually, the funnel plot of standard error by log OR was slightly asymmetric (Figure S14). This asymmetry was imputed to three potentially missing studies on the right-side of the plot, which increased the estimated effect size to 1.40 (95\% CI 0.95 to 2.05). Egger's linear regression confirmed the presence of publication bias for the analysis $(p<0.01)$, though Begg's rank correlation did not.

\subsubsection{Hospitalisation}

Meta-analysis of extracted data suggested that supplementation with ALA was not associated with an increased risk of hospitalisation ( $\mathrm{OR}=5.66,95 \% \mathrm{CI} 0.64$ to $49.85 ; p=0.119 ; \mathrm{I}^{2}=0 \%$ ) (Figure 9). The finding was robust in the leave-one-out sensitivity analysis (Figure S15). 


\section{Hospitalization}

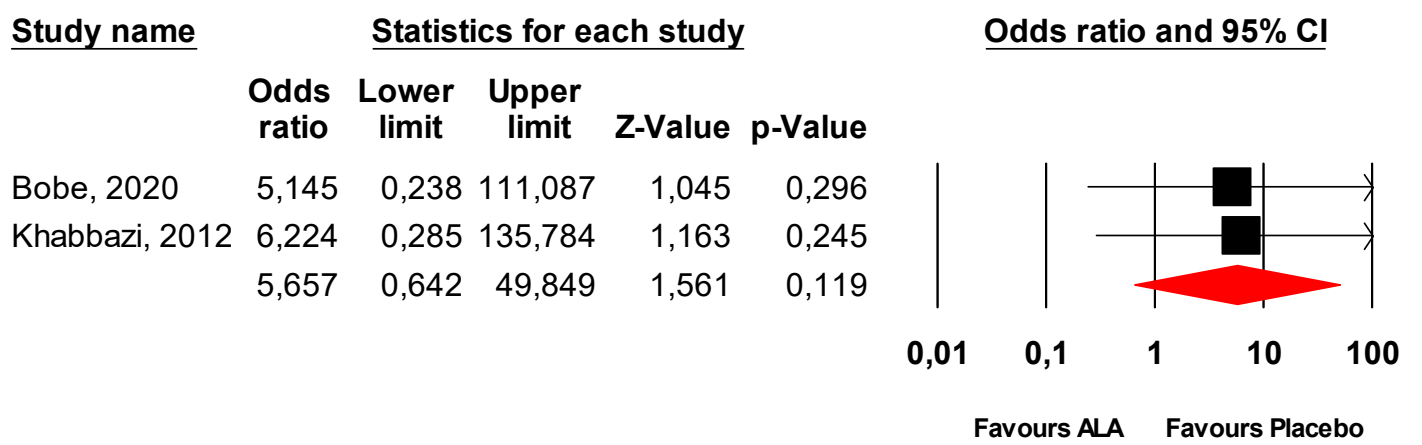

Figure 9. Forest plot for the risk of hospitalisation following ALA supplementation versus placebo.

\subsubsection{Death}

Meta-analysis of extracted data suggested that supplementation with ALA was not associated with an increased risk of death ( $\mathrm{OR}=0.56,95 \% \mathrm{CI} 0.21$ to $1.48 ; p=0.242 ; \mathrm{I}^{2}=0 \%$ ) (Figure 10). The finding was robust in the leave-one-out sensitivity analysis (Figure S16).

\section{Death}

\begin{tabular}{|c|c|c|c|c|c|c|c|c|c|}
\hline \multirow[t]{2}{*}{ Study name } & \multicolumn{5}{|c|}{ Statistics for each study } & & \multicolumn{3}{|c|}{ Odds ratio and $95 \% \mathrm{Cl}$} \\
\hline & $\begin{array}{c}\text { Odds } \\
\text { ratio }\end{array}$ & $\begin{array}{c}\text { Lower } \\
\text { limit }\end{array}$ & $\begin{array}{c}\text { Upper } \\
\text { limit }\end{array}$ & Z-Value & p-Value & & & & \\
\hline Guo, 2014 & 0,529 & 0,046 & 6,109 & $-0,510$ & 0,610 & & & & \\
\hline Hejazi, 2018 & 0,487 & 0,042 & 5,599 & $-0,577$ & 0,564 & & & & \\
\hline Khabbazi, 2012 & 0,215 & 0,010 & 4,690 & $-0,977$ & 0,328 & & - & & \\
\hline Kim, 2020 & 0,376 & 0,015 & 9,679 & $-0,590$ & 0,555 & & & & \\
\hline \multirow[t]{4}{*}{ Mohammadi, 2018} & 0,777 & 0,192 & 3,142 & $-0,354$ & 0,724 & & & & \\
\hline & 0,558 & 0,210 & 1,483 & $-1,169$ & 0,242 & & & & \\
\hline & & & & & & 0,01 & 0,1 & 10 & 100 \\
\hline & & & & & & \multicolumn{4}{|c|}{ Favours ALA Favours Placebo } \\
\hline
\end{tabular}

Figure 10. Forest plot for the risk of death following ALA supplementation versus placebo.

Visually, the funnel plot of standard error by log OR was slightly asymmetric (Figure S17). This asymmetry was imputed to three potentially missing studies on the right-side of the plot, which increased the estimated effect size to 0.71 (95\% CI 0.31 to 1.64). Egger's linear regression correlation confirmed the presence of publication bias for the analysis $(p=0.03)$, though Begg's rank correlation did not.

\subsection{Additional Analyses}

Supplementation with ALA was not associated with a significant increased risk of any AE in subsets of studies classified by smoking habit, CV disease, diabetes, pregnancy, neurological disorders, rheumatic affections, and severe renal impairment at baseline (Table 3). Furthermore, ALA supplementation was safe in children (Table 3). The findings were robust in the leave-one-out sensitivity analysis. 
Table 3. Subgroup analyses for the risk of treatment-emergent AEs, stratified by smoking habit, cardiovascular disease, presence of diabetes, pregnancy, neurological disorders, rheumatic affections, age, and severe renal impairment at baseline.

\begin{tabular}{|c|c|c|c|c|c|c|c|c|c|c|c|c|c|c|c|c|c|}
\hline & \multirow[t]{2}{*}{ AEs } & \multicolumn{2}{|c|}{ Smoking Habit } & \multicolumn{2}{|c|}{$\begin{array}{c}\text { Cardiovascular } \\
\text { Disease }\end{array}$} & \multicolumn{2}{|c|}{ Diabetes } & \multicolumn{2}{|c|}{ Pregnancy } & \multicolumn{2}{|c|}{$\begin{array}{c}\text { Neurological } \\
\text { Disorders }\end{array}$} & \multicolumn{2}{|c|}{$\begin{array}{l}\text { Rheumatic } \\
\text { Affections }\end{array}$} & \multicolumn{2}{|c|}{$\begin{array}{c}\text { Children and/or } \\
\text { Adolescents }\end{array}$} & \multicolumn{2}{|c|}{$\begin{array}{c}\text { Severe Renal } \\
\text { Impairment }\end{array}$} \\
\hline & & Yes & No & Yes & No & Yes & No & Yes & No & Yes & No & Yes & No & Yes & No & Yes & No \\
\hline \multirow{6}{*}{ 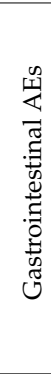 } & $\begin{array}{c}\text { Number of reported } \\
\text { AEs (active } \\
\text { arm/placebo arm) }\end{array}$ & $-/-$ & $4 / 2$ & $2 / 0$ & $97 / 88$ & $137 / 77$ & $17 / 14$ & $3 / 2$ & $180 / 97$ & $144 / 76$ & - & $5 / 2$ & $4 / 3$ & $3 / 2$ & $180 / 97$ & $-/-$ & $94 / 81$ \\
\hline & Odd ratio & - & 1.192 & 2.734 & 1.103 & 1.267 & 1.155 & 1.531 & 1.313 & 1.295 & - & 2.841 & 1.433 & 1.705 & 1.309 & - & 1.158 \\
\hline & $\begin{array}{c}95 \% \text { CI (lower limit; } \\
\text { upper limit) }\end{array}$ & - & $\begin{array}{l}0.265 \\
5.361\end{array}$ & $\begin{array}{l}0.273 \\
27.383\end{array}$ & $\begin{array}{l}0.781 ; \\
1.558\end{array}$ & $\begin{array}{l}0.879 ; \\
1.827\end{array}$ & $\begin{array}{l}0.540 \\
2.468\end{array}$ & $\begin{array}{l}0.245 ; \\
9.574\end{array}$ & $\begin{array}{l}0.966 ; \\
1.784\end{array}$ & $\begin{array}{l}0.897 \\
1.869\end{array}$ & - & $\begin{array}{r}0.500 \\
16.138\end{array}$ & $\begin{array}{l}0.300 ; \\
6.833\end{array}$ & $\begin{array}{r}0.260 \\
11.156\end{array}$ & $0.964 ; 1.779$ & - & $\begin{array}{l}0.811 ; \\
1.653\end{array}$ \\
\hline & $\mathrm{Z}$-value & - & 0.229 & 0.856 & 0.556 & 1.268 & 0.371 & 0.456 & 1.740 & 1.382 & - & 1.178 & 0.451 & 0.556 & 1.724 & - & 0.809 \\
\hline & $\mathrm{I}^{2}(\%)$ & - & 0 & 0 & 0 & 50 & 0 & 0 & 0 & 48 & - & 0 & 0 & 0 & 0 & - & 0 \\
\hline & $P$-value & - & 0.819 & 0.392 & 0.578 & 0.205 & 0.711 & 0.649 & 0.082 & 0.167 & - & 0.239 & 0.652 & 0.578 & 0.085 & - & 0.418 \\
\hline \multirow{6}{*}{ 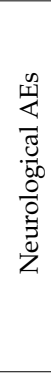 } & $\begin{array}{c}\text { Number of reported } \\
\text { AEs (active } \\
\text { arm/placebo arm) }\end{array}$ & $-/-$ & $6 / 2$ & $1 / 0$ & $19 / 18$ & $10 / 0$ & $18 / 14$ & $-/-$ & $50 / 23$ & $25 / 9$ & $-/-$ & $8 / 6$ & $0 / 1$ & $-/-$ & $50 / 23$ & $-/-$ & $22 / 16$ \\
\hline & Odd ratio & - & 1.024 & 3.078 & 1.153 & 2.368 & 1.268 & - & 1.526 & 1.718 & - & 1.474 & 0.315 & - & 1.526 & - & 3.078 \\
\hline & $\begin{array}{c}95 \% \text { CI (lower limit; } \\
\text { upper limit) }\end{array}$ & - & $\begin{array}{l}0.236 ; \\
4.442\end{array}$ & $\begin{array}{r}0.122 \\
77.905\end{array}$ & $\begin{array}{l}0.544 ; \\
2.442\end{array}$ & $\begin{array}{l}0.884 ; \\
2.634\end{array}$ & $\begin{array}{l}0.552 ; \\
2.914\end{array}$ & - & $\begin{array}{l}0.884 ; \\
2.634\end{array}$ & $\begin{array}{l}0.742 ; \\
3.977\end{array}$ & - & $\begin{array}{l}0.432 ; \\
5.027\end{array}$ & $\begin{array}{l}0.012 ; \\
7.999\end{array}$ & - & $0.884 ; 2.634$ & - & $\begin{array}{l}0.122 ; \\
77.905\end{array}$ \\
\hline & Z-value & - & 0.032 & 0.682 & 0.371 & 1.517 & 0.560 & - & 1.517 & 1.264 & - & 0.619 & -0.700 & - & 1.517 & - & 0.682 \\
\hline & $\mathrm{I}^{2}(\%)$ & - & 0 & 0 & 0 & 0 & 0 & - & 0 & 0 & - & 0 & 0 & - & 0 & - & 0 \\
\hline & $P$-value & - & 0.974 & 0.495 & 0.711 & 0.129 & 0.575 & - & 0.129 & 0.206 & - & 0.536 & 0.484 & - & 0.129 & - & 0.495 \\
\hline \multirow{6}{*}{ 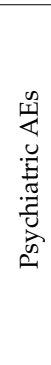 } & $\begin{array}{c}\text { Number of reported } \\
\text { AEs (active } \\
\text { arm/placebo arm) }\end{array}$ & $-/-$ & $2 / 0$ & $-/-$ & $30 / 25$ & $26 / 25$ & $4 / 0$ & $-/-$ & $30 / 25$ & $26 / 25$ & - & $-/-$ & $2 / 0$ & $-/-$ & $30 / 25$ & $-/-$ & $28 / 25$ \\
\hline & Odd ratio & - & 5.145 & - & 1.131 & 1.014 & 5.071 & - & 1.131 & 1.014 & - & - & 5.145 & - & 1.131 & - & 1.073 \\
\hline & $\begin{array}{c}95 \% \text { CI (lower limit; } \\
\text { upper limit) }\end{array}$ & - & $\begin{array}{c}0.238 \\
111.087 \\
\end{array}$ & - & $\begin{array}{l}0.644 ; \\
1.986\end{array}$ & $\begin{array}{l}0.566 ; \\
1.817\end{array}$ & $\begin{array}{r}0.582 \\
44.174 \\
\end{array}$ & - & $\begin{array}{l}0.644 ; \\
1.986\end{array}$ & $\begin{array}{l}0.566 \\
1.817\end{array}$ & - & - & $\begin{array}{c}0.238 \\
111.087\end{array}$ & - & $0.644 ; 1.986$ & - & $\begin{array}{l}0.605 ; \\
1.903\end{array}$ \\
\hline & Z-value & - & 1.045 & - & 0.429 & 0.048 & 1.470 & - & 0.429 & 0.048 & - & - & 1.045 & - & 0.429 & - & 0.242 \\
\hline & $\mathrm{I}^{2}(\%)$ & - & 0 & - & 0 & 0 & 0 & - & 0 & 0 & - & - & 0 & - & 0 & - & 0 \\
\hline & $P$-value & - & 0.296 & - & 0.668 & 0.962 & 0.142 & - & 0.668 & 0.962 & - & - & 0.296 & - & 0.668 & - & 0.809 \\
\hline
\end{tabular}


Table 3. Cont

\begin{tabular}{|c|c|c|c|c|c|c|c|c|c|c|c|c|c|c|c|c|c|}
\hline & \multirow{2}{*}{ AEs } & \multicolumn{2}{|c|}{ Smoking Habit } & \multicolumn{2}{|c|}{$\begin{array}{c}\text { Cardiovascular } \\
\text { Disease }\end{array}$} & \multicolumn{2}{|c|}{ Diabetes } & \multicolumn{2}{|c|}{ Pregnancy } & \multicolumn{2}{|c|}{$\begin{array}{c}\text { Neurological } \\
\text { Disorders }\end{array}$} & \multicolumn{2}{|c|}{$\begin{array}{l}\text { Rheumatic } \\
\text { Affections }\end{array}$} & \multicolumn{2}{|c|}{$\begin{array}{c}\text { Children and/or } \\
\text { Adolescents }\end{array}$} & \multicolumn{2}{|c|}{$\begin{array}{c}\text { Severe Renal } \\
\text { Impairment }\end{array}$} \\
\hline & & Yes & No & Yes & No & Yes & No & Yes & No & Yes & No & Yes & No & Yes & No & Yes & No \\
\hline \multirow{6}{*}{ 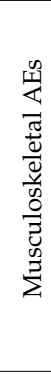 } & $\begin{array}{c}\text { Number of reported } \\
\text { AEs (active } \\
\text { arm/placebo arm) }\end{array}$ & $-/-$ & $1 / 0$ & $-/-$ & $3 / 5$ & $-/-$ & $3 / 4$ & $-/-$ & $5 / 5$ & $4 / 4$ & $-/-$ & $0 / 1$ & $1 / 0$ & $-/-$ & $5 / 5$ & $-/-$ & $3 / 5$ \\
\hline & Odd ratio & - & 3.000 & - & 0.625 & - & 0.738 & - & 0.761 & 0.683 & - & 0.321 & 3.000 & - & 0.761 & - & 0.625 \\
\hline & $\begin{array}{l}\text { 95\% CI (lower limit; } \\
\text { upper limit) }\end{array}$ & - & $\begin{array}{r}0.118 \\
76.161\end{array}$ & - & $\begin{array}{l}0.147 \\
2.661\end{array}$ & - & $\begin{array}{l}0.146 ; \\
3.723\end{array}$ & - & $\begin{array}{l}0.220 \\
2.635\end{array}$ & $\begin{array}{l}0.156 \\
2.997\end{array}$ & - & $\begin{array}{l}0.013 ; \\
8.241\end{array}$ & $\begin{array}{l}0.118 \\
76.161\end{array}$ & - & $\begin{array}{l}0.220 \\
2.635\end{array}$ & - & $\begin{array}{l}0.147 ; \\
2.661\end{array}$ \\
\hline & Z-value & - & 0.666 & - & -0.636 & - & -0.368 & - & -0.431 & -0.505 & - & -0.686 & 0.666 & - & -0.431 & - & -0.636 \\
\hline & $\mathrm{I}^{2}(\%)$ & - & 0 & - & 0 & - & 0 & - & 0 & 0 & - & 0 & 0 & - & 0 & - & 0 \\
\hline & $P$-value & - & 0.506 & - & 0.525 & - & 0.713 & - & 0.666 & 0.614 & - & 0.493 & 0.506 & - & 0.666 & - & 0.525 \\
\hline \multirow{6}{*}{ 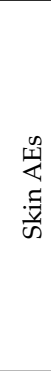 } & $\begin{array}{c}\text { Number of reported } \\
\text { AEs (active } \\
\text { arm/placebo arm) }\end{array}$ & $-/-$ & $21 / 4$ & $-/-$ & $92 / 94$ & $83 / 90$ & $14 / 6$ & $-/$ & $139 / 103$ & $83 / 91$ & $1 / 0$ & $-/-$ & $-/-$ & $-/-$ & $139 / 103$ & $2 / 0$ & $104 / 95$ \\
\hline & Odd ratio & - & 2.821 & - & 0.912 & 0.816 & 2.258 & - & 1.127 & 0.819 & 3.353 & - & - & - & 1.127 & 1.545 & 0.932 \\
\hline & $\begin{array}{c}\text { 95\% CI (lower limit; } \\
\text { upper limit) }\end{array}$ & - & $\begin{array}{l}0.899 ; \\
8.850\end{array}$ & - & $\begin{array}{l}0.635 \\
1.308\end{array}$ & $\begin{array}{l}0.559 ; \\
1.191\end{array}$ & $\begin{array}{l}0.851 ; \\
5.992\end{array}$ & - & $\begin{array}{l}0.815 ; \\
1.559\end{array}$ & $\begin{array}{l}0.563 \\
1.192\end{array}$ & $\begin{array}{r}0.120 \\
93.835\end{array}$ & - & - & - & $0.815 ; 1.559$ & $\begin{array}{r}0.067 \\
35.431\end{array}$ & $\begin{array}{l}0.653 ; \\
1.331\end{array}$ \\
\hline & Z-value & - & 1.778 & - & -0.502 & -1.052 & 1.636 & - & 0.724 & -1.041 & 0.712 & - & - & - & 0.724 & 0.272 & -0.387 \\
\hline & $\mathrm{I}^{2}(\%)$ & - & 0 & - & 29 & 0 & 0 & - & 34 & 0 & 0 & - & - & - & 34 & 0 & 36 \\
\hline & $P$-value & - & 0.075 & - & 0.616 & 0.293 & 0.102 & - & 0.469 & 0.298 & 0.477 & - & - & - & 0.469 & 0.785 & 0.699 \\
\hline \multirow{6}{*}{ 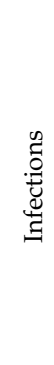 } & $\begin{array}{c}\text { Number of reported } \\
\text { AEs (active } \\
\text { arm/placebo arm) }\end{array}$ & $-/-$ & $3 / 0$ & $-/-$ & $1 / 3$ & $-/-$ & $1 / 3$ & $-/-$ & $5 / 3$ & $1 / 3$ & $-/-$ & $-/-$ & $-/-$ & $-/-$ & $5 / 3$ & $-/-$ & $4 / 3$ \\
\hline & Odd ratio & - & 3.316 & - & 0.310 & - & 0.310 & - & 0.926 & 0.310 & - & - & - & - & 0.926 & - & 0.780 \\
\hline & $\begin{array}{c}\text { 95\% CI (lower limit; } \\
\text { upper limit) }\end{array}$ & - & $\begin{array}{l}0.167 \\
65.718 \\
\end{array}$ & - & $\begin{array}{l}0.028 \\
3.364\end{array}$ & - & $\begin{array}{l}0.028 \\
3.364 \\
\end{array}$ & - & $\begin{array}{l}0.184 ; \\
4.647 \\
\end{array}$ & $\begin{array}{l}0.028 \\
3.364\end{array}$ & - & - & - & - & $0.184 ; 4.647$ & - & $\begin{array}{l}0.121 ; \\
5.028\end{array}$ \\
\hline & Z-value & - & 0.787 & - & -0.963 & - & -0.963 & - & -0.094 & -0.963 & - & - & - & - & -0.094 & - & -0.262 \\
\hline & $\mathrm{I}^{2}(\%)$ & - & 0 & - & 0 & - & 0 & - & 0 & 0 & - & - & - & - & 0 & - & 32 \\
\hline & $P$-value & - & 0.432 & - & 0.335 & - & 0.335 & - & 0.925 & 0.335 & - & - & - & - & 0.925 & - & 0.793 \\
\hline
\end{tabular}


Table 3. Cont

\begin{tabular}{|c|c|c|c|c|c|c|c|c|c|c|c|c|c|c|c|c|c|}
\hline & \multirow{2}{*}{ AEs } & \multicolumn{2}{|c|}{ Smoking Habit } & \multicolumn{2}{|c|}{$\begin{array}{l}\text { Cardiovascular } \\
\text { Disease }\end{array}$} & \multicolumn{2}{|c|}{ Diabetes } & \multicolumn{2}{|c|}{ Pregnancy } & \multicolumn{2}{|c|}{$\begin{array}{l}\text { Neurological } \\
\text { Disorders }\end{array}$} & \multicolumn{2}{|c|}{$\begin{array}{l}\text { Rheumatic } \\
\text { Affections }\end{array}$} & \multicolumn{2}{|c|}{$\begin{array}{l}\text { Children and/or } \\
\text { Adolescents }\end{array}$} & \multicolumn{2}{|c|}{$\begin{array}{c}\text { Severe Renal } \\
\text { Impairment }\end{array}$} \\
\hline & & Yes & No & Yes & No & Yes & No & Yes & No & Yes & No & Yes & No & Yes & No & Yes & No \\
\hline \multirow{6}{*}{ 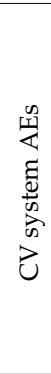 } & $\begin{array}{c}\text { Number of reported } \\
\text { AEs (active } \\
\text { arm/placebo arm) }\end{array}$ & $-/-$ & $0 / 1$ & $0 / 2$ & $71 / 53$ & $71 / 54$ & $1 / 3$ & $-/-$ & $73 / 60$ & $71 / 54$ & $-/-$ & $-/-$ & $0 / 1$ & $-/-$ & $73 / 60$ & $-/-$ & $71 / 57$ \\
\hline & Odd ratio & - & 0.149 & 0.191 & 1.441 & 1.409 & 0.450 & - & 1.247 & 1.409 & - & - & 0.333 & - & 1.247 & - & 1.313 \\
\hline & $\begin{array}{c}95 \% \text { CI (lower limit; } \\
\text { upper limit) }\end{array}$ & - & $\begin{array}{l}0.006 \\
3.733\end{array}$ & $\begin{array}{l}0.009 ; \\
4.214\end{array}$ & $\begin{array}{l}0.950 ; \\
2.186\end{array}$ & $\begin{array}{l}0.932 ; \\
2.130\end{array}$ & $\begin{array}{l}0.056 \\
3.608\end{array}$ & - & $\begin{array}{l}0.838 ; \\
1.854\end{array}$ & $\begin{array}{l}0.932 ; \\
2.130\end{array}$ & - & - & $\begin{array}{l}0.012 ; \\
9.068\end{array}$ & - & $0.838 ; 1.854$ & - & $\begin{array}{l}0.875 ; \\
1.972\end{array}$ \\
\hline & Z-value & - & -1.159 & -1.049 & 1.720 & 1.625 & -0.752 & - & 1.089 & 1.625 & - & - & -0.652 & - & 1.089 & - & 1.314 \\
\hline & $\mathrm{I}^{2}(\%)$ & - & 0 & 0 & 0 & 0 & 0 & - & 16 & 0 & - & - & 0 & - & 16 & - & 27 \\
\hline & $P$-value & - & 0.247 & 0.294 & 0.085 & 0.104 & 0.452 & - & 0.276 & 0.104 & - & - & 0.515 & - & 0.276 & - & 0.189 \\
\hline \multirow{6}{*}{ 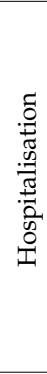 } & $\begin{array}{c}\text { Number of reported } \\
\text { AEs (active } \\
\text { arm/placebo arm) }\end{array}$ & $-/-$ & $4 / 0$ & $-/-$ & $2 / 0$ & $-/-$ & $2 / 0$ & $-/$ & $4 / 0$ & $-/-$ & - & $-/-$ & $2 / 0$ & $-/-$ & $4 / 0$ & $2 / 0$ & $2 / 0$ \\
\hline & Odd ratio & - & 5.657 & - & 5.145 & - & 5.145 & - & 5.657 & - & - & - & 5.145 & - & 5.657 & 6.224 & 5.145 \\
\hline & $\begin{array}{c}95 \% \text { CI (lower limit; } \\
\text { upper limit) }\end{array}$ & - & $\begin{array}{r}0.642 \\
49.849\end{array}$ & - & $\begin{array}{c}0.238 \\
111.087\end{array}$ & - & $\begin{array}{c}0.238 \\
111.087\end{array}$ & - & $\begin{array}{r}0.642 \\
49.849\end{array}$ & - & - & - & $\begin{array}{c}0.238 \\
111.087\end{array}$ & - & $0.642 ; 49.849$ & $\begin{array}{c}0.285 \\
135.784\end{array}$ & $\begin{array}{c}0.238 \\
111.087\end{array}$ \\
\hline & $Z$-value & - & 1.561 & - & 1.045 & - & 1.045 & - & 1.561 & - & - & - & 1.045 & - & 1.561 & 1.163 & 1.045 \\
\hline & $\mathrm{I}^{2}(\%)$ & - & 0 & - & 0 & - & 0 & - & 0 & - & - & - & 0 & - & 0 & 0 & 0 \\
\hline & $P$-value & - & 0.119 & - & 0.296 & - & 0.296 & - & 0.119 & - & - & - & 0.296 & - & 0.119 & 0.245 & 0.296 \\
\hline \multirow{6}{*}{$\begin{array}{l}\text { 营 } \\
\text { صี }\end{array}$} & $\begin{array}{l}\text { Number of reported } \\
\text { AEs (active } \\
\text { arm/placebo arm) }\end{array}$ & $-/-$ & $0 / 2$ & $4 / 5$ & $-/$ & $-/-$ & $1 / 2$ & $-/-$ & $6 / 12$ & $1 / 3$ & $-/-$ & $-/-$ & $-/-$ & $-/-$ & $6 / 12$ & $0 / 2$ & $6 / 9$ \\
\hline & Odd ratio & - & 0.215 & 0.777 & - & - & 0.529 & - & 0.558 & 0.468 & - & - & - & - & 0.558 & 0.215 & 0.657 \\
\hline & $\begin{array}{c}\text { 95\% CI (lower limit; } \\
\text { upper limit) }\end{array}$ & - & $\begin{array}{l}0.010 ; \\
4.690\end{array}$ & $\begin{array}{l}0.192 ; \\
3.142\end{array}$ & - & - & $\begin{array}{l}0.046 \\
6.109\end{array}$ & - & $\begin{array}{l}0.210 ; \\
1.483\end{array}$ & $\begin{array}{l}0.066 \\
3.300\end{array}$ & - & - & - & - & $0.210 ; 1.483$ & $\begin{array}{l}0.010 \\
4.690\end{array}$ & $\begin{array}{l}0.222 ; \\
1.947\end{array}$ \\
\hline & Z-value & - & -0.977 & -0.354 & - & - & -0.510 & - & -1.169 & -0.762 & - & - & - & - & -1.169 & -0.977 & -0.758 \\
\hline & $\mathrm{I}^{2}(\%)$ & - & 0 & 0 & - & - & 0 & - & 0 & 0 & - & - & - & - & 0 & 0 & 0 \\
\hline & $P$-value & - & 0.328 & 0.724 & - & - & 0.610 & - & 0.242 & 0.446 & - & - & - & - & 0.242 & 0.328 & 0.448 \\
\hline
\end{tabular}

AEs $=$ Adverse events; $\mathrm{CI}=$ Confidence Intervals 


\section{Discussion}

In the last years, the number of individuals assuming dietary supplements has been steadily increased worldwide $[90,91]$. Reasons for dietary supplements' use widely varies across the countries: in Europe, it is just limited to general health and well-being, while other countries permit use for medicinal purposes [92].

Considering that dietary supplement production and marketing are usually not strictly subjected to rigid rules as drugs are, there is a need for more data in order to confirm their safe use in the general population and frail subjects.

Pooling data from 71 randomized placebo-controlled clinical studies, this meta-analysis suggests that antioxidant supplementation with ALA was not associated with an increased risk of any treatment-emergent AE. Of note, statistical significance was not even achieved in subsets of studies categorized according to smoking habit, CV disease, presence of diabetes, pregnancy status, neurological disorders, rheumatic affections, renal impairment, and status of children/adolescent.

From a certain point of view, the current analysis strengthens findings from a large observational study considering outcomes data of 610 expectant mothers and their newborns that concluded ALA supplementation is safe in pregnancy even when administered at high doses [93].

These findings are particularly important because they encourage ALA use in a number of conditions in which ALA is actually proven to be effective. As a matter of fact, even though ALA supplementation has already been demonstrated to influence a broad spectrum of metabolic pathways including inflammation and glucose homeostasis [94-96], to the best of our knowledge this is the first time that ALA safety profile has been comprehensively evaluated through a pooled analysis of randomized placebo-controlled clinical studies.

Once ALA safety has been established, clinical factors for predicting treatment response should be an objective for future investigations, in order to identify the patient group that might benefit from ALA supplementation the most.

In the past, several meta-analyses showed that ALA supplementation significantly improves both positive neuropathic symptoms and neuropathic deficits to a clinically meaningful degree in diabetic patients with symptomatic polyneuropathy [97-99]. Furthermore, ALA was shown to promote weight loss in adults and obese children and adolescents [100,101].

Despite its strengths, this systematic review and meta-analysis has some limitations that mostly inherits from the included clinical studies. First, the effect size on the risk of hypoglycaemic episodes may be affected by variations in the underlying hypoglycaemic therapy in clinical trials enrolling diabetic patients. In fact, the well-recognized euglycaemic effect of ALA may require the adjustment of antidiabetic agents and insulin doses in patients taking antidiabetic drugs [101]. Second, gastrointestinal and CV system AEs included several nosological entities, justifying the probable presence of publication biases for the analysis. However, this limitation is strongly conditioned by the way the AEs were reported in the individual clinical trials. Indeed, most of the studies included in the meta-analysis report the cumulative incidence of gastrointestinal and CV system AEs, without regard to specific type of AEs. Third, AEs were difficult to identify when they were represented by exacerbations of the underlying disease for which ALA was tested (e.g., leg cramps in patients with peripheral polyneuropathy). Moreover, clinical trials testing different ALA regimens often reported the cumulative number of AEs for the supplementation versus placebo. As a result, a sub-analysis by ALA daily dose was not provided. Furthermore, different ALA formulations were tested across the included clinical studies. Despite this, heterogeneity was low for all assessed outcomes, proving that the results were reliable for the whole population and the considered sub-groups [102]. Finally, as per other dietary supplements, a relatively large number of studies have been carried out with open design and/or without a control group, so that they could not be included in a well-carried out meta-analysis.

Future research is needed to understand if sporadic adverse events associated with ALA use are related to the production quality of the used supplements, to other components of mixed supplements 
and/or to concomitant treatments or diseases, while long-term safety has been already assessed in the NATHAN (Neurological Assessment of Thioctic Acid in Diabetic Neuropathy) 1 trial [84].

\section{Conclusions}

Pooling data from the available randomized placebo-controlled clinical studies, the current meta-analysis provides data in support of the safety of the use of ALA to improve health outcomes in overall healthy individuals and in patients affected by other diseases.

Supplementary Materials: The following are available online at http://www.mdpi.com/2076-3921/9/10/1011/s1, Figure S1: Plots showing leave-one-out sensitivity analysis for the risk of gastrointestinal AEs following ALA supplementation versus placebo, Figure S2: Funnel plot detailing publication bias for the risk of gastrointestinal AEs following ALA supplementation versus placebo, Figure S3: Plot showing leave-one-out sensitivity analysis for the risk of neurological AEs following ALA supplementation versus placebo, Figure S4: Funnel plot detailing publication bias for the risk of neurological AEs following ALA supplementation versus placebo, Figure S5: Plot showing leave-one-out sensitivity analysis for the risk of psychiatric disorders following ALA supplementation versus placebo, Figure S6: Plot showing leave-one-out sensitivity analysis for the risk of musculoskeletal AEs following ALA supplementation versus placebo, Figure S7: Funnel plot detailing publication bias for the risk of musculoskeletal AEs following ALA supplementation versus placebo, Figure S8: Plot showing leave-one-out sensitivity analysis for the risk of skin AEs following ALA supplementation versus placebo, Figure S9: Funnel plot detailing publication bias for the risk of skin AEs following ALA supplementation versus placebo, Figure S10: Plot showing leave-one-out sensitivity analysis for the risk of infections following ALA supplementation versus placebo, Figure S11: Funnel plot detailing publication bias for the risk of infections following ALA supplementation versus placebo, Figure S12: Plot showing leave-one-out sensitivity analysis for the risk of CV system AEs following ALA supplementation versus placebo, Figure S13: Funnel plot detailing publication bias for the risk of CV system AEs following ALA supplementation versus placebo, Figure S14: Plot showing leave-one-out sensitivity analysis for the risk of hospitalisation following ALA supplementation versus placebo, Figure S15: Plot showing leave-one-out sensitivity analysis for the risk of death following ALA supplementation versus placebo, Figure S16: Funnel plot detailing publication bias for the risk of death following ALA supplementation versus placebo, File A: PRISMA Checklist, File B: Studies excluded from the systematic review after assessment.

Author Contributions: Conceptualization, F.F. and A.F.G.C.; methodology, F.F. and A.F.G.C.; software, F.F.; validation, F.F., M.R. and A.F.G.C.; formal analysis, F.F.; investigation, F.F., M.R., C.K. (Christoffer Krogager), C.K. (Cormac Kennedy), C.M.G.G., T.K., E.L., A.V., P.P.-M., E.F.E.W., A.Š., M.V. and A.F.G.C.; resources, F.F. and A.F.G.C.; data curation, F.F. and A.F.G.C.; writing-original draft preparation, F.F., M.R. and A.F.G.C.; writing-review and editing, C.K. (Christoffer Krogager), C.K. (Cormac Kennedy), C.M.G.G., T.K., E.L., A.V., P.P.-M., E.F.E.W., A.Š. and M.V.; visualization, F.F. and A.F.G.C.; supervision, A.F.G.C.; project administration, F.F. and A.F.G.C. All authors have read and agreed to the published version of the manuscript.

Funding: This research received no external funding.

Conflicts of Interest: MR is currently Chief Medical and Scientific Advisor, Novo Nordisk South East Europe, Middle East and Africa (SEEMEA). The other authors declare no conflict of interest.

\section{References}

1. Tibullo, D.; Li Volti, G.; Giallongo, C.; Grasso, S.; Tomassoni, D.; Anfuso, C.D.; Lupo, G.; Amenta, F.; Avola, R.; Bramanti, V. Biochemical and clinical relevance of alpha lipoic acid: Antioxidant and anti-inflammatory activity, molecular pathways and therapeutic potential. Inflamm. Res. 2017, 66,947-959. [CrossRef] [PubMed]

2. Biewenga, G.P.; Haenen, G.R.; Bast, A. The pharmacology of the antioxidant lipoic acid. Gen. Pharmacol. 1997, 29, 315-331. [CrossRef]

3. Pershadsingh, H.A. Alpha-lipoic acid: Physiologic mechanisms and indications for the treatment of metabolic syndrome. Expert Opin. Investig. Drugs 2007, 16, 291-302. [CrossRef] [PubMed]

4. Rahmanabadi, A.; Mahboob, S.; Amirkhizi, F.; Hosseinpour-Arjmand, S.; Ebrahimi-Mameghani, M. Oral $\alpha$-lipoic acid supplementation in patients with non-alcoholic fatty liver disease: Effects on adipokines and liver histology features. Food Funct. 2019, 10, 4941-4952. [CrossRef] [PubMed]

5. Hosseinpour-Arjmand, S.; Amirkhizi, F.; Ebrahimi-Mameghani, M. The effect of alpha-lipoic acid on inflammatory markers and body composition in obese patients with non-alcoholic fatty liver disease: A randomized, double-blind, placebo-controlled trial. J. Clin. Pharm. Ther. 2019, 44, 258-267. [CrossRef] [PubMed] 
6. Yukina, M.; Nuralieva, N.; Solovyev, M.; Troshina, E.; Vasilyev, E. Insulin autoimmune syndrome. Endocrinol. Diabetes Metab. Case Rep. 2020, 2020, 19-0159. [CrossRef]

7. Izzo, V.; Greco, C.; Corradini, D.; Infante, M.; Staltari, M.T.; Romano, M.; Bellia, A.; Lauro, D.; Uccioli, L. Insulin autoimmune syndrome in an Argentine woman taking $\alpha$-lipoic acid: A case report and review of the literature. SAGE Open Med. Case Rep. 2018, 6, 2050313X18819601. [CrossRef]

8. Bresciani, E.; Bussi, A.; Bazzigaluppi, E.; Balestrieri, G. Insulin autoimmune syndrome induced by $\alpha$-lipoic acid in a Caucasian woman: Case report. Diabetes Care. 2011, 34, e146. [CrossRef]

9. Gatti, M.; Ippoliti, I.; Poluzzi, E.; Antonazzo, I.C.; Moro, P.A.; Moretti, U.; Menniti-Ippolito, F.; Mazzanti, G.; De Ponti, F.; Raschi, E. Assessment of adverse reactions to $\alpha$-lipoic acid containing dietary supplements through spontaneous reporting systems. Clin. Nutr. 2020. E-pub ahead of print. [CrossRef]

10. Moher, D.; Liberati, A.; Tetzlaff, J.; Altman, D.G.; PRISMA Group. Preferred reporting items for systematic reviews and meta-analyses: The PRISMA statement. BMJ 2009, 339, b2535. [CrossRef]

11. Higgins, J.; Green, S. Cochrane Handbook for Systematic Reviews of Interventions; Version 5.0. 2. 2009; John Wiley and Sons Ltd: Chichester, UK, 2010.

12. Fogacci, F.; Ferri, N.; Toth, P.P.; Ruscica, M.; Corsini, A.; Cicero, A.F.G. Efficacy and Safety of Mipomersen: A Systematic Review and Meta-Analysis of Randomized Clinical Trials. Drugs 2019, 79, 751-766. [CrossRef] [PubMed]

13. Guyatt, G.H.; Oxman, A.D.; Vist, G.E.; Kunz, R.; Falck-Ytter, Y.; Alonso-Coello, P.; Schünemann, H.J.; GRADE Working Group. GRADE: An emerging consensus on rating quality of evidence and strength of recommendations. BMJ 2008, 336, 924-926. [CrossRef]

14. Borenstein, M.; Hedges, L.; Higgins, J.; Rothstein, H. Comprehensive Meta-Analysis Version 3; Biostat: Englewood, NJ, USA, 2005; Volume 104.

15. Higgins, J.P.T.; Thomas, J.; Chandler, J.; Cumpston, M.; Li, T.; Page, M.J.; Welch, V.A. Cochrane Handbook for Systematic Reviews of Interventions; Version 6.0 (updated July 2019); Cochrane: London, UK, 2019; Available online: www.training.cochrane.org/handbook (accessed on 15 September 2020).

16. Melsen, W.G.; Bootsma, M.C.; Rovers, M.M.; Bonten, M.J. The effects of clinical and statistical heterogeneity on the predictive values of results from meta-analyses. Clin. Microbiol. Infect. 2004, 20, 123-129. [CrossRef] [PubMed]

17. Haenszel, W.; Hon, N.B. Statistical approaches to the study of cancer with particular reference to case registers. J. Chronic. Dis. 1956, 4, 589-599. [CrossRef]

18. Fogacci, S.; Fogacci, F.; Banach, M.; Michos, E.D.; Hernandez, A.V.; Lip, G.Y.H.; Blaha, M.J.; Toth, P.P.; Borghi, C.; Cicero, A.F.G. Lipid and Blood Pressure Meta-analysis Collaboration (LBPMC) Group. Vitamin D supplementation and incident preeclampsia: A systematic review and meta-analysis of randomized clinical trials. Clin. Nutr. 2020, 39, 1742-1752. [CrossRef]

19. Fogacci, F.; Banach, M.; Mikhailidis, D.P.; Bruckert, E.; Toth, P.P.; Watts, G.F.; Reiner, Ž.; Mancini, J.; Rizzo, M.; Mitchenko, O.; et al. Safety of red yeast rice supplementation: A systematic review and meta-analysis of randomized controlled trials. Pharmacol. Res. 2019, 143, 1-16. [CrossRef]

20. Ahmadi, A.; Mazooji, N.; Roozbeh, J.; Mazloom, Z.; Hasanzade, J. Effect of alpha-lipoic acid and vitamin E supplementation on oxidative stress, inflammation, and malnutrition in hemodialysis patients. Iran J. Kidney Dis. 2013, 7, 461-467. [PubMed]

21. Ansar, H.; Mazloom, Z.; Kazemi, F.; Hejazi, N. Effect of alpha-lipoic acid on blood glucose, insulin resistance and glutathione peroxidase of type 2 diabetic patients. Saudi Med. J. 2011, 32, 584-588.

22. Aslfalah, H.; Jamilian, M.; Rafiei, F.; Khosrowbeygi, A. Reduction in maternal serum values of glucose and gamma-glutamyltransferase after supplementation with alpha-lipoic acid in women with gestational diabetes mellitus. J. Obstet. Gynaecol. Res. 2019, 45, 313-317. [CrossRef]

23. Aslfalah, H.; Jamilian, M.; Khosrowbeygi, A. Elevation of the adiponectin/leptin ratio in women with gestational diabetes mellitus after supplementation with alpha-lipoic acid. Gynecol. Endocrinol. 2019, 35, 271-275. [CrossRef]

24. Baumgartner, S.; Mensink, R.P.; Haenen, G.R.; Bast, A.; Binder, C.J.; Bekers, O.; Husche, C.; Lütjohann, D.; Plat, J. The effects of vitamin E or lipoic acid supplementation on oxyphytosterols in subjects with elevated oxidative stress: A randomized trial. Sci. Rep. 2017, 7, 15288. [CrossRef] [PubMed] 
25. Baziar, N.; Nasli-Esfahani, E.; Djafarian, K.; Qorbani, M.; Hedayati, M.; Mishani, M.A.; Faghfoori, Z.; Ahmaripour, N.; Hosseini, S. The Beneficial Effects of Alpha Lipoic Acid Supplementation on Lp-PLA2 Mass and Its Distribution between HDL and apoB-Containing Lipoproteins in Type 2 Diabetic Patients: A Randomized, Double-Blind, Placebo-Controlled Trial. Oxid. Med. Cell Longev. 2020, 2020, 5850865. [CrossRef] [PubMed]

26. Bobe, G.; Michels, A.J.; Zhang, W.J.; Purnell, J.Q.; Woffendin, C.; Pereira, C.; Vita, J.A.; Thomas, N.O.; Traber, M.G.; Frei, B.; et al. A Randomized Controlled Trial of Long-Term (R)- $\alpha$-Lipoic Acid Supplementation Promotes Weight Loss in Overweight or Obese Adults without Altering Baseline Elevated Plasma Triglyceride Concentrations. J. Nutr. 2020, 150, 2336-2345. [CrossRef] [PubMed]

27. Boriani, F.; Granchi, D.; Roatti, G.; Merlini, L.; Sabattini, T.; Baldini, N. Alpha-lipoic Acid After Median Nerve Decompression at the Carpal Tunnel: A Randomized Controlled Trial. J. Hand Surg. Am. 2017, 42, 236-242. [CrossRef] [PubMed]

28. Carbone, M.; Pentenero, M.; Carrozzo, M.; Ippolito, A.; Gandolfo, S. Lack of efficacy of alpha-lipoic acid in burning mouth syndrome: A double-blind, randomized, placebo-controlled study. Eur. J. Pain 2009, 13, 492-496. [CrossRef]

29. Cavalcanti, D.R.; da Silveira, F.R. Alpha lipoic acid in burning mouth syndrome-A randomized double-blind placebo-controlled trial. J. Oral Pathol. Med. 2009, 38, 254-261. [CrossRef]

30. Durastanti, V.; Tinelli, E.; Di Rezze, S.; Berardelli, A.; Millefiorini, E. Alpha lipoic acid as add-on therapy to subcutaneous interferon $\beta$-1a for relapsing-remitting multiple sclerosis: A pilot study. IJABPT 2016, 7 , 336-341.

31. El Amrousy, D.; El-Afify, D. Effects of alpha lipoic acid as a supplement in obese children and adolescents. Cytokine 2020, 130, 155084. [CrossRef]

32. Falardeau, J.; Fryman, A.; Wanchu, R.; Marracci, G.H.; Mass, M.; Wooliscroft, L.; Bourdette, D.N.; Murchison, C.F.; Hills, W.L.; Yadav, V. Oral lipoic acid as a treatment for acute optic neuritis: A blinded, placebo controlled randomized trial. Mult. Scler. J. Exp. Transl. Clin. 2019, 5, 2055217319850193. [CrossRef]

33. Femiano, F.; Scully, C. Burning mouth syndrome (BMS): Double blind controlled study of alpha-lipoic acid (thioctic acid) therapy. J. Oral Pathol. Med. 2002, 31, 267-269. [CrossRef] [PubMed]

34. Georgakouli, K.; Fatouros, I.G.; Fragkos, A.; Tzatzakis, T.; Deli, C.K.; Papanikolaou, K.; Koutedakis, Y.; Jamurtas, A.Z. Exercise and Redox Status Responses Following Alpha-Lipoic Acid Supplementation in G6PD Deficient Individuals. Antioxidants 2018, 7, 162. [CrossRef]

35. Gianturco, V.; Bellomo, A.; D’Ottavio, E.; Formosa, V.; Iori, A.; Mancinella, M.; Troisi, G.; Marigliano, V. Impact of therapy with alpha-lipoic acid (ALA) on the oxidative stress in the controlled NIDDM: A possible preventive way against the organ dysfunction? Arch. Gerontol. Geriatr. 2009, 49, 129-133. [CrossRef] [PubMed]

36. Gilron, I.; Robb, S.; Tu, D.; Holden, R.; Towheed, T.; Ziegler, D.; Wang, L.; Milev, R.; Gray, C. Double-blind, randomized, placebo-controlled crossover trial of alpha-lipoic acid for the treatment of fibromyalgia pain: The IMPALA trial. Pain 2020. [CrossRef] [PubMed]

37. Gosselin, L.E.; Chrapowitzky, L.; Rideout, T.C. Metabolic effects of $\alpha$-lipoic acid supplementation in pre-diabetics: A randomized, placebo-controlled pilot study. Food Funct. 2019, 10, 5732-5738. [CrossRef] [PubMed]

38. Guo, Y.; Jones, D.; Palmer, J.L.; Forman, A.; Dakhil, S.R.; Velasco, M.R.; Weiss, M.; Gilman, P.; Mills, G.M.; Noga, S.J.; et al. Oral alpha-lipoic acid to prevent chemotherapy-induced peripheral neuropathy: A randomized, double-blind, placebo-controlled trial. Support. Care Cancer 2014, 22, 1223-1231. [CrossRef] [PubMed]

39. Haghighian, H.K.; Haidari, F.; Mohammadi-Asl, J.; Dadfar, M. Randomized, triple-blind, placebo-controlled clinical trial examining the effects of alpha-lipoic acid supplement on the spermatogram and seminal oxidative stress in infertile men. Fertil. Steril. 2015, 104, 318-324. [CrossRef]

40. Hejazi, N.; Mazloom, Z.; Zand, F.; Rezaianzadeh, A.; Nikandish, R. The Beneficial Effects of $\alpha$-Lipoic Acid in Critically Ill Patients: A Prospective, Randomized, Double-Blind, Placebo-Controlled Trial. Asian J. Anesthesiol. 2018, 56, 45-55.

41. Huang, E.A.; Gitelman, S.E. The effect of oral alpha-lipoic acid on oxidative stress in adolescents with type 1 diabetes mellitus. Pediatr. Diabetes 2008, 9, 69-73. [CrossRef] 
42. Huerta, A.E.; Prieto-Hontoria, P.L.; Sáinz, N.; Martínez, J.A.; Moreno-Aliaga, M.J. Supplementation with $\alpha$-Lipoic Acid Alone or in Combination with Eicosapentaenoic Acid Modulates the Inflammatory Status of Healthy Overweight or Obese Women Consuming an Energy-Restricted Diet. J. Nutr. 2016, 146. [CrossRef]

43. Huerta, A.E.; Navas-Carretero, S.; Prieto-Hontoria, P.L.; Martínez, J.A.; Moreno-Aliaga, M.J. Effects of $\alpha$-lipoic acid and eicosapentaenoic acid in overweight and obese women during weight loss. Obesity 2015, 23, 313-321. [CrossRef]

44. Jacob, S.; Ruus, P.; Hermann, R.; Tritschler, H.J.; Maerker, E.; Renn, W.; Augustin, H.J.; Dietze, G.J.; Rett, K. Oral administration of RAC-alpha-lipoic acid modulates insulin sensitivity in patients with type-2 diabetes mellitus: A placebo-controlled pilot trial. Free Radic. Biol. Med. 1999, 27, 309-314. [CrossRef]

45. Jamshidi, K.; Abdollahzad, H.; Nachvak, M.; Rezaei, M.; Golpayegani, M.R.; Sharifi Zahabi, E. Effects of Alpha-Lipoic Acid Supplementation on Cardiovascular Disease Risk Factors in $\beta$-Thalassemia Major Patients: A Clinical Trial Crossover Study. J. Blood Med. 2020, 11, 131-139. [CrossRef]

46. Jariwalla, R.J.; Lalezari, J.; Cenko, D.; Mansour, S.E.; Kumar, A.; Gangapurkar, B.; Nakamura, D. Restoration of blood total glutathione status and lymphocyte function following alpha-lipoic acid supplementation in patients with HIV infection. J. Altern. Complement Med. 2008, 14, 139-146. [CrossRef]

47. Khabbazi, T.; Mahdavi, R.; Safa, J.; Pour-Abdollahi, P. Effects of alpha-lipoic acid supplementation on inflammation, oxidative stress, and serum lipid profile levels in patients with end-stage renal disease on hemodialysis. J. Ren. Nutr. 2012, 22, 244-250. [CrossRef] [PubMed]

48. Khalili, M.; Soltani, M.; Moghadam, S.A.; Dehghan, P.; Azimi, A.; Abbaszadeh, O. Effect of alpha-lipoic acid on asymmetric dimethylarginine and disability in multiple sclerosis patients: A randomized clinical trial. Electron. Physician 2017, 9, 4899-4905. [CrossRef] [PubMed]

49. Khalili, M.; Azimi, A.; Izadi, V.; Eghtesadi, S.; Mirshafiey, A.; Sahraian, M.A.; Motevalian, A.; Norouzi, A.; Sanoobar, M.; Eskandari, G.; et al. Does lipoic acid consumption affect the cytokine profile in multiple sclerosis patients: A double-blind, placebo-controlled, randomized clinical trial. Neuroimmunomodulation 2014, 21, 291-296. [CrossRef] [PubMed]

50. Kim, B.J.; Hunter, A.; Brucker, A.J.; Hahn, P.; Gehrs, K.; Patel, A.; Edwards, A.O.; Li, Y.; Khurana, R.N.; Nissim, I.; et al. Orally Administered Alpha Lipoic Acid as a Treatment for Geographic Atrophy: A Randomized Clinical Trial. Ophthalmol. Retin. 2020, 4, 889-898. [CrossRef] [PubMed]

51. Kim, N.W.; Song, Y.M.; Kim, E.; Cho, H.S.; Cheon, K.A.; Kim, S.J.; Park, J.Y. Adjunctive $\alpha$-lipoic acid reduces weight gain compared with placebo at 12 weeks in schizophrenic patients treated with atypical antipsychotics: A double-blind randomized placebo-controlled study. Int. Clin. Psychopharmacol. 2016, 31, 265-274. [CrossRef] [PubMed]

52. Koh, E.H.; Lee, W.J.; Lee, S.A.; Kim, E.H.; Cho, E.H.; Jeong, E.; Kim, D.W.; Kim, M.S.; Park, J.Y.; Park, K.G.; et al. Effects of alpha-lipoic Acid on body weight in obese subjects. Am. J. Med. 2011, 124, 85.e1-85.e8. [CrossRef]

53. Lampitella, A.; Rossi, E.; Carrino, F.; Griffo, P.; Carrino, R. Effetto dell'acido lipoico e della vitamina E sulla polineuropatia diabetica: Analisi statistica. Prog. Nutr. 2005, 7, 116-134. (In Italian)

54. Lee, S.J.; Jeong, S.J.; Lee, Y.C.; Lee, Y.H.; Lee, J.E.; Kim, C.H.; Min, K.W.; Cha, B.Y. Effects of High-Dose $\alpha$-Lipoic Acid on Heart Rate Variability of Type 2 Diabetes Mellitus Patients with Cardiac Autonomic Neuropathy in Korea. Diabetes Metab. J. 2017, 41, 275-283. [CrossRef] [PubMed]

55. Loy, B.D.; Fling, B.W.; Horak, F.B.; Bourdette, D.N.; Spain, R.I. Effects of lipoic acid on walking performance, gait, and balance in secondary progressive multiple sclerosis. Complement Ther. Med. 2018, 41, 169-174. [CrossRef] [PubMed]

56. López-D'alessandro, E.; Escovich, L. Combination of alpha lipoic acid and gabapentin, its efficacy in the treatment of Burning Mouth Syndrome: A randomized, double-blind, placebo controlled trial. Med. Oral Patol. Oral Cir. Bucal. 2011, 16, e635-e640. [CrossRef] [PubMed]

57. López-Jornet, P.; Camacho-Alonso, F.; Leon-Espinosa, S. Efficacy of alpha lipoic acid in burning mouth syndrome: A randomized, placebo-treatment study. J. Oral Rehabil. 2009, 36, 52-57. [CrossRef] [PubMed]

58. Magis, D.; Ambrosini, A.; Sándor, P.; Jacquy, J.; Laloux, P.; Schoenen, J. A randomized double-blind placebo-controlled trial of thioctic acid in migraine prophylaxis. Headache 2007, 47, 52-57. [CrossRef] [PubMed]

59. Manning, P.J.; Sutherland, W.H.; Williams, S.M.; Walker, R.J.; Berry, E.A.; De Jong, S.A.; Ryalls, A.R. The effect of lipoic acid and vitamin E therapies in individuals with the metabolic syndrome. Nutr. Metab. Cardiovasc. Dis. 2013, 23, 543-549. [CrossRef] 
60. Marfella, R.; Barbieri, M.; Sardu, C.; Rizzo, M.R.; Siniscalchi, M.; Paolisso, P.; Ambrosino, M.; Fava, I.; Materazzi, C.; Cinquegrana, G.; et al. Effects of $\alpha$-lipoic acid therapy on sympathetic heart innervation in patients with previous experience of transient takotsubo cardiomyopathy. J. Cardiol. 2016, 67, 153-161. [CrossRef]

61. Marshall, A.W.; Graul, R.S.; Morgan, M.Y.; Sherlock, S. Treatment of alcohol-related liver disease with thioctic acid: A six month randomised double-blind trial. Gut 1982, 23, 1088-1093. [CrossRef]

62. Martins, V.D.; Manfredini, V.; Peralba, M.C.; Benfato, M.S. Alpha-lipoic acid modifies oxidative stress parameters in sickle cell trait subjects and sickle cell patients. Clin. Nutr. 2009, 28, 192-197. [CrossRef]

63. Mendes, P.R.; Félix Ddos, S.; Silva, P.C.; Pereira, G.H.; Simões, M.O. Effect of alpha lipoic acid on the blood cell count and iron kinetics in hypertensive patients. Nutr. Hosp. 2014, 31, 883-889.

64. Mendoza-Núñez, V.M.; García-Martínez, B.I.; Rosado-Pérez, J.; Santiago-Osorio, E.; Pedraza-Chaverri, J.; Hernández-Abad, V.J. The Effect of $600 \mathrm{mg}$ Alpha-lipoic Acid Supplementation on Oxidative Stress, Inflammation, and RAGE in Older Adults with Type 2 Diabetes Mellitus. Oxid. Med. Cell Longev. 2019, 2019, 3276958. [CrossRef] [PubMed]

65. Mirtaheri, E.; Pourghassem Gargari, B.; Kolahi, S.; Asghari-Jafarabadi, M.; Hajaliloo, M. Effect of alpha-lipoic acid supplementation on serum lipid profile in women with rheumatoid arthritis. NFSR 2014, 1, 11-18.

66. Mohammadi, V.; Khorvash, F.; Feizi, A.; Askari, G. Does Alpha-lipoic Acid Supplementation Modulate Cardiovascular Risk Factors in Patients with Stroke? A Randomized, Double-blind Clinical Trial. Int. J. Prev. Med. 2018, 9, 34. [PubMed]

67. Mohammadi, V.; Khalili, M.; Eghtesadi, S.; Dehghani, S.; Jazayeri, S.; Aghababaee, S.K.; Sabour, H.; Saberi, H.; Eghtesadi, M.; Gohari, M.R. The effect of alpha-lipoic acid (ALA) supplementation on cardiovascular risk factors in men with chronic spinal cord injury: A clinical trial. Spinal Cord. 2015, 53, 621-624. [CrossRef] [PubMed]

68. Mollo, R.; Zaccardi, F.; Scalone, G.; Scavone, G.; Rizzo, P.; Navarese, E.P.; Manto, A.; Pitocco, D.; Lanza, G.A.; Ghirlanda, G.; et al. Effect of $\alpha$-lipoic acid on platelet reactivity in type 1 diabetic patients. Diabetes Care 2012, 35, 196-197. [CrossRef]

69. Monroy Guízar, E.A.; García Benavides, L.; Ambriz Plascencia, A.R.; Pascoe González, S.; Totsuka Sutto, S.E.; Cardona Muñoz, E.G.; Méndez-Del Villar, M. Effect of Alpha-Lipoic Acid on Clinical and Neurophysiologic Recovery of Carpal Tunnel Syndrome: A Double-Blind, Randomized Clinical Trial. J. Med. Food 2018, 21, 521-526. [CrossRef]

70. Palacios-Sánchez, B.; Moreno-López, L.A.; Cerero-Lapiedra, R.; Llamas-Martínez, S.; Esparza-Gómez, G. Alpha lipoic acid efficacy in burning mouth syndrome. A controlled clinical trial. Med. Oral Patol. Oral Cir. Bucal. 2015, 20, e435-e440. [CrossRef]

71. Porasuphatana, S.; Suddee, S.; Nartnampong, A.; Konsil, J.; Harnwong, B.; Santaweesuk, A. Glycemic and oxidative status of patients with type 2 diabetes mellitus following oral administration of alpha-lipoic acid: A randomized double-blinded placebo-controlled study. Asia Pac. J. Clin. Nutr. 2012, 21, 12-21.

72. Pourghasem Gargari, B.; Kolahi, S.; Dehghan, P.; Khabbazi, A.; Mirtaheri, E. Effects of alpha-lipoic acid supplementation on clinical status and anthropometric indices in women with rheumatoid arthritis. Curr. Top. Nutraceutical Res. 2015, 13, 33-40.

73. Ruhnau, K.J.; Meissner, H.P.; Finn, J.R.; Reljanovic, M.; Lobisch, M.; Schütte, K.; Nehrdich, D.; Tritschler, H.J.; Mehnert, H.; Ziegler, D. Effects of 3-week oral treatment with the antioxidant thioctic acid (alpha-lipoic acid) in symptomatic diabetic polyneuropathy. Diabetic Med. 1999, 16, 1040-1043. [CrossRef]

74. Safa, J.; Ardalan, M.R.; Rezazadehsaatlou, M.; Mesgari, M.; Mahdavi, R.; Jadid, M.P. Effects of alpha lipoic acid supplementation on serum levels of IL-8 and TNF- $\alpha$ in patient with ESRD undergoing hemodialysis. Int. Urol. Nephrol. 2014, 46, 1633-1638. [CrossRef] [PubMed]

75. Sammour, H.; Elkholy, A.; Rasheedy, R.; Fadel, E. The effect of alpha lipoic acid on uterine wound healing after primary cesarean section: A triple-blind placebo-controlled parallel-group randomized clinical trial. Arch. Gynecol. Obstet. 2019, 299, 665-673. [CrossRef] [PubMed]

76. Sardu, C.; Santulli, G.; Santamaria, M.; Barbieri, M.; Sacra, C.; Paolisso, P.; D’Amico, F.; Testa, N.; Caporaso, I.; Paolisso, G.; et al. Effects of Alpha Lipoic Acid on Multiple Cytokines and Biomarkers and Recurrence of Atrial Fibrillation Within 1 Year of Catheter Ablation. Am. J. Cardiol. 2017, 119, 1382-1386. [CrossRef] 
77. Scaramuzza, A.; Giani, E.; Redaelli, F.; Ungheri, S.; Macedoni, M.; Giudici, V.; Bosetti, A.; Ferrari, M.; Zuccotti, G.V. Alpha-Lipoic Acid and Antioxidant Diet Help to Improve Endothelial Dysfunction in Adolescents with Type 1 Diabetes: A Pilot Trial. J. Diabetes Res. 2015, 2015, 474561. [CrossRef] [PubMed]

78. Sola, S.; Mir, M.Q.; Cheema, F.A.; Khan-Merchant, N.; Menon, R.G.; Parthasarathy, S.; Khan, B.V. Irbesartan and lipoic acid improve endothelial function and reduce markers of inflammation in the metabolic syndrome: Results of the Irbesartan and Lipoic Acid in Endothelial Dysfunction (ISLAND) study. Circulation 2005, 111, 343-348. [CrossRef] [PubMed]

79. Spain, R.; Powers, K.; Murchison, C.; Heriza, E.; Winges, K.; Yadav, V.; Cameron, M.; Kim, E.; Horak, F.; Simon, J.; et al. Lipoic acid in secondary progressive MS: A randomized controlled pilot trial. Neurol. Neuroimmunol. Neuroinflamm. 2017, 4, e374. [CrossRef] [PubMed]

80. Sun, Y.D.; Dong, Y.D.; Fan, R.; Zhai, L.L.; Bai, Y.L.; Jia, L.H. Effect of (R)- $\alpha$-lipoic acid supplementation on serum lipids and antioxidative ability in patients with age-related macular degeneration. Ann. Nutr. Metab. 2012, 60, 293-297. [CrossRef]

81. Tromba, L.; Perla, F.M.; Carbotta, G.; Chiesa, C.; Pacifico, L. Effect of Alpha-Lipoic Acid Supplementation on Endothelial Function and Cardiovascular Risk Factors in Overweight/Obese Youths: A Double-Blind, Placebo-Controlled Randomized Trial. Nutrients 2019, 11, 375. [CrossRef] [PubMed]

82. Udupa, A.; Nahar, P.; Shah, S.; Kshirsagar, M.; Ghongane, B. A comparative study of effects of omega-3 Fatty acids, alpha lipoic Acid and vitamin e in type 2 diabetes mellitus. Ann. Med. Health Sci. Res. 2013, 3, 442-446. [PubMed]

83. Vincent, H.K.; Bourguignon, C.M.; Vincent, K.R.; Taylor, A.G. Effects of alpha-lipoic acid supplementation in peripheral arterial disease: A pilot study. J. Altern. Complement Med. 2007, 13, 577-584. [CrossRef] [PubMed]

84. Yadav, V.; Marracci, G.; Lovera, J.; Woodward, W.; Bogardus, K.; Marquardt, W.; Shinto, L.; Morris, C.; Bourdette, D. Lipoic acid in multiple sclerosis: A pilot study. Mult. Scler. 2005, 11, 159-165. [CrossRef] [PubMed]

85. Yan, W.; Li, N.; Hu, X.; Huang, Y.; Zhang, W.; Wang, Q.; Wang, F.; Wang, C.; Zhai, X.; Xu, R.; et al. Effect of oral ALA supplementation on oxidative stress and insulin sensitivity among overweight/obese adults: A double-blinded, randomized, controlled, cross-over intervention trial. Int. J. Cardiol. 2013, 167, 602-603. [CrossRef] [PubMed]

86. Zembron-Lacny, A.; Gajewski, M.; Naczk, M.; Dziewiecka, H.; Siatkowski, I. Physical activity and alpha-lipoic acid modulate inflammatory response through changes in thiol redox status. J. Physiol. Biochem. 2013, 69, 397-404. [CrossRef] [PubMed]

87. Zembron-Lacny, A.; Slowinska-Lisowska, M.; Szygula, Z.; Witkowski, K.; Szyszka, K. The comparison of antioxidant and hematological properties of $\mathrm{N}$-acetylcysteine and alpha-lipoic acid in physically active males. Physiol. Res. 2009, 58, 855-861.

88. Ziegler, D.; Low, P.A.; Litchy, W.J.; Boulton, A.J.; Vinik, A.I.; Freeman, R.; Samigullin, R.; Tritschler, H.; Munzel, U.; Maus, J.; et al. Efficacy and safety of antioxidant treatment with $\alpha$-lipoic acid over 4 years in diabetic polyneuropathy: The NATHAN 1 trial. Diabetes Care 2011, 34, 2054-2060. [CrossRef]

89. Ziegler, D.; Ametov, A.; Barinov, A.; Dyck, P.J.; Gurieva, I.; Low, P.A.; Munzel, U.; Yakhno, N.; Raz, I.; Novosadova, M.; et al. Oral treatment with alpha-lipoic acid improves symptomatic diabetic polyneuropathy: The SYDNEY 2 trial. Diabetes Care 2006, 29, 2365-2370. [CrossRef]

90. Mahady, G.B. Global harmonization of herbal health claims. J. Nutr. 2001, 131, 1120S-1123S. [CrossRef]

91. Thakkar, S.; Anklam, E.; Xu, A.; Ulberth, F.; Li, J.; Li, B.; Hugas, M.; Sarma, N.; Crerar, S.; Swift, S.; et al. Regulatory landscape of dietary supplements and herbal medicines from a global perspective. Regul. Toxicol. Pharmacol. 2020, 114, 104647. [CrossRef]

92. Han, T.; Bai, J.; Liu, W.; Hu, Y. A systematic review and meta-analysis of $\alpha$-lipoic acid in the treatment of diabetic peripheral neuropathy. Eur. J. Endocrinol. 2012, 167, 465-471. [CrossRef]

93. Parente, E.; Colannino, G.; Picconi, O.; Monastra, G. Safety of oral alpha-lipoic acid treatment in pregnant women: A retrospective observational study. Eur. Rev. Med. Pharmacol. Sci. 2017, 21, 4219-4227.

94. Rahimlou, M.; Asadi, M.; Banaei Jahromi, N.; Mansoori, A. Alpha-lipoic acid (ALA) supplementation effect on glycemic and inflammatory biomarkers: A Systematic Review and meta- analysis. Clin. Nutr. ESPEN 2019, 32, 16-28. [CrossRef] [PubMed] 
95. Tabrizi, R.; Borhani-Haghighi, A.; Mirhosseini, N.; Lankarani, K.B.; Naghibzadeh-Tahami, A.; Akbari, M.; Heydari, S.T.; Sangari, M.; Kolahdooz, F.; Raygan, F.; et al. The effects of alpha-lipoic acid supplementation on fasting glucose and lipid profiles among patients with stroke: A systematic review and meta-analysis of randomized controlled trials. J. Diabetes Metab. Disord. 2019, 18, 585-595. [CrossRef]

96. Akbari, M.; Ostadmohammadi, V.; Lankarani, K.B.; Tabrizi, R.; Kolahdooz, F.; Khatibi, S.R.; Asemi, Z. The effects of alpha-lipoic acid supplementation on glucose control and lipid profiles among patients with metabolic diseases: A systematic review and meta-analysis of randomized controlled trials. Metabolism 2018, 87, 56-69. [CrossRef] [PubMed]

97. Eisenberg, D.M.; Davis, R.B.; Ettner, S.L.; Appel, S.; Wilkey, S.; Van Rompay, M.; Kessler, R.C. Trends in alternative medicine use in the United States, 1990-1997: Results of a follow-up national survey. JAMA 1998, 280, 1569-1575. [CrossRef] [PubMed]

98. Mijnhout, G.S.; Kollen, B.J.; Alkhalaf, A.; Kleefstra, N.; Bilo, H.J. Alpha lipoic Acid for symptomatic peripheral neuropathy in patients with diabetes: A meta-analysis of randomized controlled trials. Int. J. Endocrinol. 2012, 2012, 456279. [CrossRef] [PubMed]

99. Ziegler, D.; Nowak, H.; Kempler, P.; Vargha, P.; Low, P.A. Treatment of symptomatic diabetic polyneuropathy with the antioxidant alpha-lipoic acid: A meta-analysis. Diabet Med. 2004, 21, 114-121. [CrossRef]

100. Vajdi, M.; Abbasalizad Farhangi, M. Alpha-lipoic acid supplementation significantly reduces the risk of obesity in an updated systematic review and dose response meta-analysis of randomised placebo-controlled clinical trials. Int. J. Clin. Pract. 2020, 74, e13493. [CrossRef]

101. Ebada, M.A.; Fayed, N.; Fayed, L.; Alkanj, S.; Abdelkarim, A.; Farwati, H.; Hanafy, A.; Negida, A.; Ebada, M.; Noser, Y. Efficacy of Alpha-lipoic Acid in The Management of Diabetes Mellitus: A Systematic Review and Meta-analysis. Iran J. Pharm. Res. 2019, 18, 2144-2156.

102. Sandercock, P. The authors say: 'The data are not so robust because of heterogeneity'—So, how should I deal with this systematic review? Meta-analysis and the clinician. Cerebrovasc. Dis. 2011, 31, 615-620. [CrossRef]

Publisher's Note: MDPI stays neutral with regard to jurisdictional claims in published maps and institutional affiliations.

(C) 2020 by the authors. Licensee MDPI, Basel, Switzerland. This article is an open access article distributed under the terms and conditions of the Creative Commons Attribution (CC BY) license (http://creativecommons.org/licenses/by/4.0/). 